\title{
Feasibility Study for Future Spaceborne Coherent Doppler Wind Lidar, Part 2: Measurement Simulation Algorithms and Retrieval Error Characterization
}

\author{
Philippe BARON, Shoken ISHII \\ Applied Electromagnetic Research Institute, National Institute of Information and Communications Technology, \\ Koganei, Japan \\ Kozo OKAMOTO \\ Meteorological Research Institute, Japan Meteorological Agency, Tsukuba, Japan \\ Kyoka GAMO \\ Fujitsu FIP Corporation, Tokyo, Japan \\ Kohei MIZUTANI \\ Applied Electromagnetic Research Institute, National Institute of Information and Communications Technology, \\ Koganei, Japan \\ Chikako TAKAHASHI \\ Fujitsu FIP Corporation, Tokyo, Japan \\ Toshikazu ITABE \\ Applied Electromagnetic Research Institute, National Institute of Information and Communications Technology, \\ Koganei, Japan \\ Toshiki IWASAKI \\ Graduate School of Science, Tohoku University, Miyagi, Japan
}

Takuji KUBOTA

Earth Observation Research Center, Japan Aerospace Exploration Agency, Tsukuba, Japan

Takashi MAKI

Meteorological Research Institute, Japan Meteorological Agency, Tsukuba, Japan

\footnotetext{
Corresponding author: Philippe Baron, Applied Electromagnetic Research Institute, National Institute of Information and Communications Technology, 4-2-1, NukuiKitamachi, Koganei 184-8795, Japan

E-mail: baron@nict.go.jp

J-stage Advance Published Date: 19 June 2017

(C)2017, Meteorological Society of Japan
} 


\title{
Riko OKI
}

Earth Observation Research Center, Japan Aerospace Exploration Agency, Tsukuba, Japan

\section{Satoshi OCHIAI}

Applied Electromagnetic Research Institute, National Institute of Information and Communications Technology, Koganei, Japan

\section{Daisuke SAKAIZAWA}

Earth Observation Research Center, Japan Aerospace Exploration Agency, Tsukuba, Japan

\section{Masaki SATOH}

The University of Tokyo, Kashiwa, Japan

Yohei SATOH

Satellite Technology Innovation Office, Japan Aerospace Exploration Agency, Tsukuba, Japan

Taichu Y. TANAKA

Meteorological Research Institute, Japan Meteorological Agency, Tsukuba, Japan

and

\section{Motoaki YASUI}

Social Innovation Unit Strategic Program Produce Office, National Institute of Information and Communications Technology, Koganei, Japan

(Manuscript received 6 December 2016, in final form 6 June 2017)

\begin{abstract}
A feasibility study of tropospheric wind measurements using a coherent Doppler lidar aboard a super low altitude satellite is being conducted in Japan. The considered lidar uses a $2.05 \mu \mathrm{m}$ laser light source of $3.75 \mathrm{~W}$. In order to assess the measurement performances, simulations of wind measurements were conducted. The mission definition is presented in a companion paper (Part 1) while, in this paper, we describe the measurement simulator and characterize the errors on the retrieved line-of-sight (LOS) winds. Winds are retrieved from the Doppler-shift of the noisy backscattered signal with a horizontal resolution of $100 \mathrm{~km}$ along the orbit track and a vertical resolution between 0.5 and $2 \mathrm{~km}$. Cloud and wind fields are the pseudo-truth of an Observing System Simulation Experiment while aerosol data are from the Model-of-Aerosol-Species-IN-the-Global-AtmospheRe (MASINGAR) constrained with the pseudo-truth wind. We present the results of the analysis of a full month of data in summer time for a near-polar orbiting satellite and a LOS nadir angle of $35^{\circ}$. Below $\approx 8 \mathrm{~km}$, the ratio of good retrievals is $30-55 \%$ and the median LOS wind error is better than $0.6 \mathrm{~m} \mathrm{~s}^{-1}\left(1.04 \mathrm{~m} \mathrm{~s}^{-1}\right.$ for the horizontal wind). In the upper troposphere, the ratio is less than $15 \%$ in the southern hemisphere and high-latitudes. However, the ratio is still $35 \%$ in the northern Tropics and mid-latitudes where ice-clouds frequently occur. The upper-tropospheric median LOS-wind measurement error is between 1-2 m s$~^{-1}$ depending on the latitude $\left(1.74-3.5 \mathrm{~m} \mathrm{~s}^{-1}\right.$ for the horizontal wind). These errors are dominated by uncertainties induced by spatial atmospheric inhomogeneities.
\end{abstract}

Keywords Doppler lidar; satellite; remote sensing; wind; aerosol; simulation 


\section{Introduction}

The only spaceborne wind profile measurements currently available have been performed using passive remote sensing techniques, and their altitude range is limited to the middle and upper atmosphere above 30 $\mathrm{km}$ (Baron et al. 2013). In order to address the strong demand for global observations in the troposphere (Baker et al. 2014), the European Space Agency (ESA) plans to launch the Atmospheric Dynamics Mission Aeolus equipped with a direct-detection lidar at $\lambda=$ $355 \mathrm{~nm}$ (Stoffelen et al. 2005). In this mission, only a single component of the wind vector will be profiled to demonstrate the feasibility and benefits of such systems. Future missions are under consideration to profile the full horizontal wind vector.

In the United States, systems under investigation use either coherent (heterodyne) or direct-detection methods at $\lambda=2 \mu \mathrm{m}$ and $\lambda=355 \mathrm{~nm}$, respectively (Baker et al. 1995; Emmitt 2001; Baker et al. 2014; Singh et al. 2014). A mission combining both techniques is also considered in order to cumulate the advantages of each method. More specifically, the wavelength and the narrow spectral bandwidth of the coherent infrared Doppler wind lidar (IR-DWL) are well suited for the detection of the aerosol backscattered signal in the lower troposphere but, the available bandwidth is not sufficient to detect the molecular signal. On the other hand, the possibility of detecting molecular signals using direct detection gives such methods more sensitivity at higher altitudes.

In Japan, our working group is studying a spaceborne coherent IR-DWL (Ishii et al. 2016) and has developed laser technologies at $\lambda=1.6$ and $2 \mu \mathrm{m}$ (Ishii et al. 2012). In order to support the definition of the mission, we conduct simulations using the Integrated Satellite Observation SIMulator for Coherent Doppler Lidar (ISOSIM) with the characteristics of a $2.05 \mu \mathrm{m}$ laser which is the more mature technology at the current stage of this study (Ishii et al. 2017). The output of ISOSIM consists of simulated wind measurements along the line-of-sight (LOS). These are subsequently used in an Observing System Simulation Experiment (OSSE) (Okamoto et al. 2010) to quantitatively evaluate the impact of the observation parameters on the retrievals, as well as the potential of such a mission for improving atmospheric models.

The aim of this paper is to describe ISOSIM, to characterize the wind retrieval errors and to assess the measurement performances from simulated data. The details about the mission definition, hardware development, scientific objectives and OSSE are given in companion papers (Ishii et al. 2016, 2017; Okamoto et al. 2017). In Section 2, the measurement principles are described. Section 3 presents ISOSIM with a special focus on the atmospheric data. The algorithms for simulating the backscattered signal and retrieving the LOS wind velocity are described in Section 4. In that section, the retrieval errors are characterized under ideal conditions with known and constant atmospheric parameters. In Section 5, the instrument performance under realistic conditions is assessed using ISOSIM calculations from August 2010. The summary and concluding remarks are given in the last section.

\section{Measurement principles}

\subsection{Measurement characteristics}

The objective of the feasibility study is to define a mission capable of profiling the two horizontal components of the tropospheric winds with a precision better than $1 \mathrm{~m} \mathrm{~s}^{-1}$ and a spatial resolution better than $100 \mathrm{~km}$ along the orbit track and between 0.5 and $2 \mathrm{~km}$ in height (Ishii et al. 2016, 2017). The two components of the horizontal wind are constructed from quasi simultaneous and collocated LOS wind measurements using two detectors pointing at perpendicular azimuth angles of $45^{\circ}$ and $135^{\circ}$. The nadir angle is set to $35^{\circ}$ which provides a good compromise between the inclination of the LOS and the distance between the instrument and target range (Ishii et al. 2017). This entails a precision on the LOS wind of $\approx$ $0.5 \mathrm{~m} \mathrm{~s}^{-1}$.

We assume that the line-of-sight angles (nadir, azimuth) are known with enough precision to mitigate to a satisfactory degree the Doppler shift induced by the satellite velocity $\left(\approx 4 \mathrm{~km} \mathrm{~s}^{-1}\right.$ along the LOS for an azimuth angle of $45^{\circ}$ ). For example, a systematic error of $0.1 \mathrm{MHz}$ on the satellite-velocity Doppler-shift is achieved if the uncertainties on the nadir and azimuth angles are 21 and $37 \mu \mathrm{rad}$, respectively (Frehlich 2001). The use of the Next-Generation Star Tracker (NSTT) (Matsumoto et al. 2013) should allow us to know the LOS angles with an error consistent with that needed for wind measurements at a rate of $4 \mathrm{~Hz}$.

The Super-Low-Altitude Test Satellite (180-220 $\mathrm{km}$ ) under development by the Japan Aerospace Exploration Agency (JAXA) is considered as a platform (Kawasaki et al. 2013). Hence, the laser power requirement can be reduced by a factor of 4-12 compared with that needed with traditional low orbit platforms (400-700 km). We will learn more about the platform stability at such low altitudes after the first test flight planned by JAXA in 2017.

The LOS wind velocity is derived from the differ- 
ence between the backscattered signal and the transmitted pulse laser frequencies. The so-called Doppler frequency shift is

$$
F_{d}=2 \frac{v}{\lambda},
$$

where $\lambda$ is the laser incident pulse wavelength, $F_{d}$ is the Doppler frequency $(\mathrm{Hz})$ and $v$ is the LOS velocity $\left(\mathrm{m} \mathrm{s}^{-1}\right)$. Only the LOS wind measurements are discussed in this manuscript since it is the parameter used in the OSSE. Also to avoid any confusion between LOS and horizontal winds, the results are given as Doppler-shift frequencies. At the wavelength of $2 \mu \mathrm{m}$, they are simply related as follows:

$$
F_{d, \mathrm{MHz}} \approx v,
$$

with $F_{d, \mathrm{MHz}}$ the Doppler frequency expressed in MHz.

The detector mixes the return signal with a reference laser beam (local-oscillator) with a known frequency to produce a radio-frequency (RF) signal that beats at a frequency related to the Doppler shift. The return signal is sampled at the frequency $F_{s}=400$ $\mathrm{MHz}$ (time interval of $2.5 \mathrm{~ns}$ ) and the receiver bandwidth is

$$
B=F_{s} / 2=200 \mathrm{MHz} \text {. }
$$

The transmitted pulse and reference laser frequencies are separated by $100 \mathrm{MHz}$. Hence, the frequency of a signal with no Doppler-shift is in the middle of the bandwidth (100 MHz) and Doppler frequencies between $\pm 100 \mathrm{MHz}$ can be measured.

The instrument characteristics are summarized in Table 1. They are similar to those of existing instruments (Ishii et al. 2010, 2012; Iwai et al. 2013). The laser power is $3.75 \mathrm{~W}$, the pulse repetition frequency $\mathrm{PRF}=30 \mathrm{~Hz}$ and the pulse energy $E_{p}=125 \mathrm{~mJ}$. The full-width at half-maximum (FWHM) of the laser pulse power profile is $\tau=200 \mathrm{~ns}$. More details about the hardware characteristics are discussed in (Ishii et al. 2017).

\subsection{Signal to noise ratio}

Throughout the manuscript, the signal power is normalized so that the detector-noise power is 1 and the total power is equal to the wideband signal-to-noise ratio (SNR). The SNR is that of a single pulse without measurement accumulation.

The SNR is proportional to the power of the atmospheric signal $\mathrm{P}_{L}\left(\mathrm{~W} \mathrm{~m}^{-2}\right)$ if the detector noise is dominated by the local-oscillator shot noise (Frehlich and Yadlowsky 1994; Henderson et al. 2005; Henderson

\begin{tabular}{|c|c|}
\hline \multicolumn{2}{|l|}{ Laser $\lambda=2.1 \mu \mathrm{m}$} \\
\hline Frequency $\Leftrightarrow$ velocity & $1.0 \mathrm{~m} \mathrm{~s}^{-1} \equiv 1.0 \mathrm{MHz}$ \\
\hline Pulse energy & $125 \mathrm{~mJ}$ \\
\hline Peak power & $0.625 \mathrm{MW}$ \\
\hline Frequency repetition (PRF) & $30 \mathrm{~Hz}$ \\
\hline Pulse power profile FWHM $(\tau)$ & $200 \mathrm{~ns}$ \\
\hline Power spectrum STD $\left(w_{p}\right)$ & $0.9370 \mathrm{MHz}$ \\
\hline LO frequency precision & $0.1 \mathrm{MHz}$ \\
\hline Detector quantum efficiency $\left(\eta_{q}\right)$ & 0.8 \\
\hline Optics efficiency $\left(\eta_{o}\right)$ & 0.25 \\
\hline Heterodyne efficiency $\left(\eta_{H}\right)$ & 0.4 \\
\hline \multicolumn{2}{|l|}{ Range gate characteristics } \\
\hline Sampling frequency $\left(F_{s}\right)$ & $400 \mathrm{MHz}$ \\
\hline Number of samples per range gate $(\mathrm{M})$ & 256 \\
\hline range gate duration / vertical resolution & $640 \mathrm{~ns} / 78.6 \mathrm{~m}$ \\
\hline Independent data $(\Omega)$ & 0.60 \\
\hline \multicolumn{2}{|l|}{ Observation characteristics } \\
\hline Telescope diameter & $40 \mathrm{~cm}$ \\
\hline Satellite altitude & 200 to $220 \mathrm{~km}$ \\
\hline Nadir angle & $35^{\circ}$ \\
\hline Satellite velocity wrt surface & $7.7 \mathrm{~km} \mathrm{~s}^{-1}$ \\
\hline $\begin{array}{l}\text { Pulses accumulation for } 100 \mathrm{~km} \\
\text { along-track range }\end{array}$ & $\approx 390$ \\
\hline
\end{tabular}
2013). It is derived as follows:
Table 1. Instrumental and observational characteristics.

\begin{tabular}{lr}
\hline Retrieval layers (altitude wrt surface / resolution) \\
Layers $1-6$ & $0.1-3 \mathrm{~km} / \approx 0.5 \mathrm{~km}$ \\
Layers $7-11$ & $3-8 \mathrm{~km} / 1 \mathrm{~km}$ \\
Layers $12-17$ & $8-20 \mathrm{~km} / 2 \mathrm{~km}$
\end{tabular}

$$
\begin{aligned}
& \operatorname{SNR}(t)= \frac{\left\langle s^{2}(t)\right\rangle}{\left\langle\Xi^{2}(t)\right\rangle}=\eta_{q} \frac{\lambda \mathrm{P}_{L}(t)}{B h c} \\
&=K\left(B, E_{p}\right)\left[\int_{r_{0}}^{r_{s}}\left(\frac{A\left(t^{\prime}-t\right) T\left(r^{\prime}\right)}{r^{\prime}}\right)^{2} \beta\left(r^{\prime}\right) d r^{\prime}\right. \\
& \\
&\left.+\frac{A_{b}}{\pi}\left(\frac{A\left(t_{s}-t\right) T\left(r_{s}\right)}{r_{s}}\right)^{2}\right]
\end{aligned}
$$

where

$$
K\left(B, E_{p}\right)=\frac{\eta_{q} \lambda}{B h c} \eta_{H} \eta_{o} A_{s} E_{p} .
$$

In these equations, $s(t)$ is proportional to the electric field of the backscattered radiation, $\Xi(t)$ is the instrument (shot) noise, $E_{p}$ is the transmitted pulse energy $(\mathrm{J}), t$ is the time of the measurement $(t=0$ when the laser pulse is shot), $r^{\prime}=c t^{\prime} / 2$ is a position on the LOS ( $r^{\prime}=0$ is the lidar position), $r_{s}=c t_{s} / 2$ is the distance to the Earth's surface, and $r_{0}$ is the distance to the top of the atmosphere (here set at $30 \mathrm{~km}$ ). The antenna 
area is $A_{s}\left(\mathrm{~m}^{2}\right), B$ is the frequency bandwidth (Eq. 3) and $h=6.6261 \times 10^{-34} \mathrm{~J} \mathrm{~s}$ is the Planck constant. The weighting function $A^{2}(t)$ is the profile of the transmitted pulse power $\left(\int A^{2}(t) d t=1\right), \beta$ is the aerosol backscatter coefficient $\left(\mathrm{m}^{-1} \mathrm{sr}^{-1}\right), T$ is the one-way transmission between the target $r$ and the instrument and $A_{b}$ is the surface albedo. The parameters $\eta_{q}$ and $\eta_{o}$ are the quantum and optical efficiencies, and $\eta_{H}$ is the heterodyne efficiency. The sensitivity of $\eta_{H}$ to refractive turbulence is neglected for far range observation at infrared wavelength (Wu et al. 2013). Here it is assumed that the illuminated air-mass is fully captured by the receiver field-of-view, the refraction is negligible and the surface reflection is Lambertian (Kiemle et al. 2011).

The single-shot SNR can be approximated as follows:

$$
\mathrm{SNR} \approx 40\left(\frac{400}{\Delta_{H, k m}}\right)^{2} \beta_{a t, k m} .
$$

where $\Delta_{H, k m}$ is the altitude difference (expressed in $\mathrm{km}$ ) between the lidar and range gate, and $\beta_{a t t, k m}$ is the backscatter coefficient expressed in $\mathrm{km}^{-1} \mathrm{sr}^{-1}$ and averaged over the range-gate. Typical values of $\beta_{a t t, k m}$ at $\lambda=2 \mu \mathrm{m}$, are between $10^{-7}-10^{-2} \mathrm{~km}^{-1} \mathrm{sr}^{-1}$ (Henderson et al. 2005). This approximation is not used in ISOSIM but useful for quick conversions between SNR and backscatter coefficients.

\subsection{Speckle noise}

Figure 1 shows a synthetic RF signal computed using the same model as that implemented in ISOSIM (Section 4.1) with a constant SNR $=4$ over a time range of $1200 \mathrm{~ns}$. The SNR is large enough to highlight fast oscillations induced by the Doppler shift (50 $\mathrm{MHz}$ ) as well as intensity modulation due to the socalled speckle noise. The speckles are interference patterns produced by randomly distributed backscatterers illuminated by the laser pulse (Dabas et al. 1994). Their size corresponds to the range resolution of the measurement which is, at first approximation, the 1/e FWHM of the signal autocorrelation function (See Appendix 3):

$$
\Delta_{c}=2.4 \frac{c \tau}{2}=72 \mathrm{~m}
$$

where $c=2.9979 \times 10^{8} \mathrm{~m} \mathrm{~s}^{-1}$ is the speed of light in vacuum. An optimized signal analysis requires the use of at least all samples within one speckle which is the longest coherent time series in the signal. The smallest

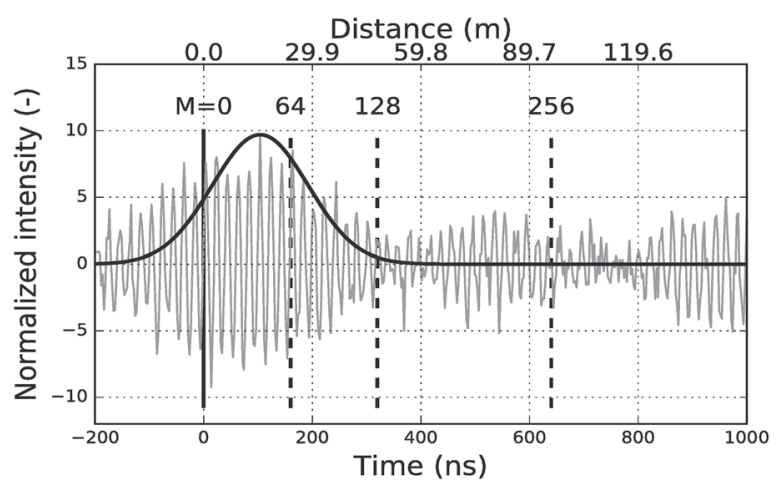

Fig. 1. Synthetic RF signal with a sampling frequency $F_{s}=400 \mathrm{MHz}$, a Doppler frequency of $F_{d}=50 \mathrm{MHz}$ and an $\mathrm{SNR}=4$. The thick black line is a Gaussian shape function with a FWHM $\tau=200 \mathrm{~ns}$ identical to that of the transmitted pulse power profile. The ranges for $M=64,128$ and 256 samples are shown.

range gates considered in this study are made of $M=$ 256 samples $\left(F_{s}=400 \mathrm{MHz}\right)$ which correspond to 1.33 speckles (Eq. 48 in Appendix 3).

\section{ISOSIM}

\subsection{Overview}

ISOSIM simulates all laser shots transmitted to the atmosphere from the satellite. Each LOS is divided into range gates of $M=256$ samples (78.6 m vertical thickness) in which the atmospheric parameters are constant. A time domain RF signal is generated for each range gate, independent of each other.

Power spectra are computed for each narrow range gate and averaged to match the retrieval spatial resolutions. The Doppler frequency is then retrieved from each average spectrum. Hereafter, we will use the term "narrow-range" to qualify the properties of range gates of the 256 samples (e.g., narrow-range frequency).

The characteristics of the Earth's surface are taken into account. The elevation with respect to the geoid is described on a $30^{\prime \prime}$ grid derived from the Shuttle Radar Topography Mission (SRTM) (version 7) (Becker et al. 2009). The surface albedo is either $A_{b}=0.2$ for continents or 0.02 for seas and oceans. The Global SelfConsistent Hierarchical High-Resolution Shoreline (GSHHS) database (Wessel and Smith 1996) is used to identify the surface types.

A single orbit (about $90 \mathrm{mn}$ ) contains $162 \times 10^{3}$ shots and $\sim 35$ narrow-range gates per shot. Considering a homogeneous atmosphere over narrow-ranges 
allows us to use an efficient algorithm to calculate the time-domain signal which is necessary to cope with the large number of computations. Such a model is, however, not well suited for taking into account the sharp termination of the laser path at the Earth's surface, and a future version should include a better algorithm for the range near the surface (Salamitou et al. 1995; Frehlich 1997).

\subsection{Atmospheric data}

The atmospheric properties are derived from 3-D fields of the horizontal wind, temperature, pressure, aerosol concentration, the total water content and cloud coverage. The vertical wind that is small compared to the horizontal wind is not taken into account. The total water content includes the amount of water in liquid and ice phases. The parameters are taken from the OSSE pseudo-truth except for the aerosols concentration which are taken from the version 3 of the Model of Aerosol Species IN the Global Atmo-
spheRe (MASINGAR) (Tanaka et al. 2003) driven with the pseudo-truth wind and sea surface temperature. The horizontal resolution is $1.125^{\circ} \times 1.125^{\circ}$ and the profiles are defined on 48 and 60 vertical levels for MASINGAR and the pseudo-truth, respectively. The vertical sampling typically increases from 20 m near the surface to $1 \mathrm{~km}$ in the lower stratosphere. The data are given every hour and linearly interpolated at the measurement times.

Aerosols are classified into 5 types of particles, namely organic and black carbons, mineral dust, sea salt and sulfate. The attenuated backscatter coefficients are calculated with the Mie scattering theory using the parameters summarized in Tables 2 and 3. The hygroscopic growth is taken into account using the MASINGAR parametrization originally taken from (Chin et al. 2002). Figure 2 shows the zonal median of the aerosol backscatter coefficients at $2.05 \mu \mathrm{m}$. The highest values are found in the lower atmosphere (below $3 \mathrm{~km}$ ) of the Tropics and the sub-Tropics. Over

Table 2. Aerosol and cloud particle characteristics in MASINGAR. The aerosol wet radius growth factors are given for relative humidity of 50 , $70,80,90,95$ and $99 \%$, respectively.

\begin{tabular}{|c|c|}
\hline Dust & $\begin{array}{l}\text { Concentration in } 10 \text { radius bins from } 0.1-10 \mu \mathrm{m} * \\
\text { Density: } 2.5 \mathrm{~g} \mathrm{~cm}^{-3}\end{array}$ \\
\hline Sea salt & $\begin{array}{l}\text { Concentration in } 10 \text { radius bins from } 0.1-10 \mu \mathrm{m}^{*} \\
\text { Density: } 2.25 \mathrm{~g} \mathrm{~cm}^{-3} \\
\text { Wet radius growth factors: } 1.6,1.8,2.0,2.4,2.9,4.8\end{array}$ \\
\hline Black carbon & $\begin{array}{l}\text { Total concentration, log-normal size distribution. } \\
\text { Radius range from } 0.01 \text { to } 1 \mu \mathrm{m} \text { (100 ranges) } \\
\text { Density: } 1.00 \mathrm{~g} \mathrm{~cm}^{-3} \\
\text { Standard deviation: } 1.55 \\
\text { Median dry radius: } 0.0118 \mu \mathrm{m} \\
\text { Wet radius growth factors: } 1 ., 1 ., 1.2,1.4,1.5,1.9\end{array}$ \\
\hline Organic carbon & $\begin{array}{l}\text { Total concentration }\left(\mathrm{m}^{-3}\right), \text { log-normal size distribution. } \\
\text { Radius range from } 0.01 \text { to } 20 \mu \mathrm{m} \\
\text { Density: } 1.8 \mathrm{~g} \mathrm{~cm}^{-3} \\
\text { Standard deviation: } 2.20 \\
\text { Median dry radius: } 0.0212 \mu \mathrm{m} \\
\text { Wet radius growth factors: } 1.2,1.4,1.5,1.6,1.8,2.2\end{array}$ \\
\hline Sulfate & $\begin{array}{l}\text { Total concentration, log-normal size distribution. } \\
\text { Radius range from } 0.01 \text { to } 1 \mu \mathrm{m} \text { (100 ranges) } \\
\text { Standard deviation: } 2.03 \\
\text { Density: } 1.77 \mathrm{~g} \mathrm{~cm}^{-3} \\
\text { Median dry radius: } 0.0695 \mu \mathrm{m} \\
\text { Wet radius growth factors: } 1.4,1.5,1.6,1.8,1.9,2.2\end{array}$ \\
\hline Cloud particle & Total concentration of equivalent liquid water \\
\hline
\end{tabular}

* The volume-mean radii for the bins are $0.136,0.215,0.340,0.540,0.855$, $1.355,2.148,3.405,5.396,8.553 \mu \mathrm{m}$. 
Table 3. Dry aerosol and water refractive indexes (Hess et al. 1998). For a given relative humidity, the aerosol refractive index is $G^{-3} n_{d}+\left(1-G^{-3}\right) n_{l}$, with $G$ the relative humidity dependent radius growth factor (Table 2), $n_{d}$ and $n_{l}$ are the values of the refractive indexes of the dry aerosol and of the liquid water, respectively.

\begin{tabular}{lll}
\hline \multicolumn{1}{c}{ Type } & \multicolumn{1}{c}{$1.0 \mu \mathrm{m}$} & \multicolumn{1}{c}{$2.0 \mu \mathrm{m}$} \\
\hline Dust & $1.53-4 \times 10^{-3} i$ & $1.53-7.6 \times 10^{-3} i$ \\
Sea salt & $1.47-1.41 \times 10^{-4} i$ & $1.45-10^{-3} i$ \\
Black carbon $^{-3}$ & $1.76-4.43 \times 10^{-1} i$ & $1.80-4.92 \times 10^{-1} i$ \\
Organic carbon $^{1}$ & $1.63-0.70 \times 10^{-3} i$ & $1.63-0.70 \times 10^{-3} i$ \\
Sulfate & $1.42-1.53 \times 10^{-6} i$ & $1.384-2.26 \times 10^{-3} i$ \\
Liquid water & $1.63-1.9 \times 10^{-6} i$ & $1.306-1.1 \times 10^{-3} i$ \\
Ice & $1.30-2.12 \times 10^{-6} i$ & $1.270-1.582 \times 10^{-3} i$ \\
\hline
\end{tabular}

${ }^{1}$ from http://www.met.reading.ac.uk/adient/

the continental regions, the backscatter coefficients are between $10^{-5}-10^{-4} \mathrm{~km}^{-1} \mathrm{sr}^{-1}$ which correspond to SNR values of about $10^{-3}-10^{-2}$ for the $2.05 \mu \mathrm{m}$ lidar at a height of $200 \mathrm{~km}$ (Fig. 3). Over the maritime regions, the values strongly decrease with altitudes from about $10^{-4} \mathrm{~km}^{-1} \mathrm{sr}^{-1}\left(\mathrm{SNR}=10^{-2}\right.$ ) near the surface to less than $10^{-5} \mathrm{~km}^{-1} \mathrm{sr}^{-1}\left(\mathrm{SNR}=10^{-3}\right)$ near a height of $3 \mathrm{~km}$.

For most of the cases in the mid and upper troposphere, the backscatter coefficients are less than $10^{-5}$ $\mathrm{km}^{-1} \mathrm{sr}^{-1}\left(\mathrm{SNR}<10^{-3}\right)$. Near 30-40N, the backscatter coefficients are enhanced between 5 and $15 \mathrm{~km}$ (values near $10^{-5} \mathrm{~km}^{-1} \mathrm{sr}^{-1}$ ) due to a strong lift of desert dust aerosols.

The use of aerosol data from the previous MASINGAR version (v2) leads to significantly larger attenuated backscatter coefficients by a factor of about 10 (Fig. S1a in Ishii et al. 2016). The primary difference between versions 2 and 3, is the treatment of sea-salt aerosol whose mass concentration is significantly decreased in the newest version. Also, the wet deposition parameter in $\mathrm{v} 3$ is stronger than that in v2, which further reduces aerosol concentration in the troposphere. However in spite of these differences, the wind measurement performances estimated using v2 and v3 do not show significant differences (see Section 5.3).

A proper evaluation of MASINGAR aerosol concentration is out of the scope of this analysis. Most of the comparisons between MASINGAR and observations are for the boundary layers, aerosol optical depth (AOD) or lidar observations in East Asia (Sekiyama et al. 2010; Uchino et al. 2017). Comparisons with satellite AOD observation by MODIS $(550 \mathrm{~nm})$ sug-

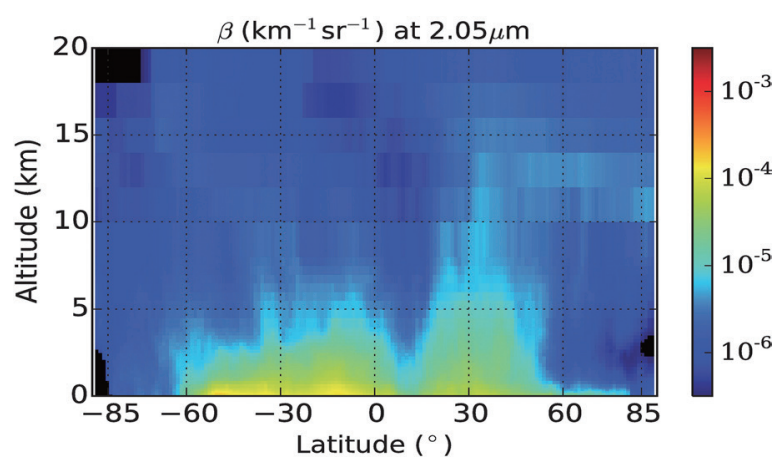

Fig. 2. Zonal median vertical distribution of the aerosol attenuated backscatter coefficients at $2 \mu \mathrm{m}$ derived from MASINGAR (v3) on 1st August 2008.

gests that the mineral dust from the Sahara Desert, and anthropogenic aerosols from China and India are underestimated, while the biomass burning aerosols in South America and Africa are relatively in good agreement (Private communication from MASINGAR team). So, it is important to keep in mind that there are large uncertainties on the aerosol data especially in the mid and upper troposphere. In a previous study (Wu et al. 2013), spaceborne DWL performances were assessed using aerosol $2 \mu \mathrm{m}$ backscatter coefficients derived from CALIPSO observations at $532 \mathrm{~nm}$. A comparison of this dataset with ours has to be done with caution since the former is a yearly climatology, only representative of clear sky conditions and derived from observations at $0.532 \mu \mathrm{m}$ which are not sensitive to aerosols in the same way as that at $2 \mu \mathrm{m}$. However, the median values derived from CALIPSO measurements are in-between those computed with MASINGAR v2 and v3, and consequently, these datasets should be considered consistent.

Clouds are randomly generated along the orbit track on a grid with a transversal width of $100 \mathrm{~km}$ and horizontal and vertical spacings of 10 and $0.2 \mathrm{~km}$, respectively. The probability of generating cloud particles on a grid point is given by the cloud coverage ratio from the pseudo-truth (Fig. 4). It is worth to note that atmospheric models are expected to underestimate the cloud coverage (Cesana and Chepfer 2012; Chepfer et al. 2012). The number of particles is derived from the equivalent liquid water content. We consider that the particles are in a liquid state if the temperature is above $0{ }^{\circ} \mathrm{C}$ and in ice state if the temperature is below $-40{ }^{\circ} \mathrm{C}$ (Cesana and Chepfer 2013). In the transition range from $-40{ }^{\circ} \mathrm{C}$ to $0{ }^{\circ} \mathrm{C}$, both regimes are contin- 


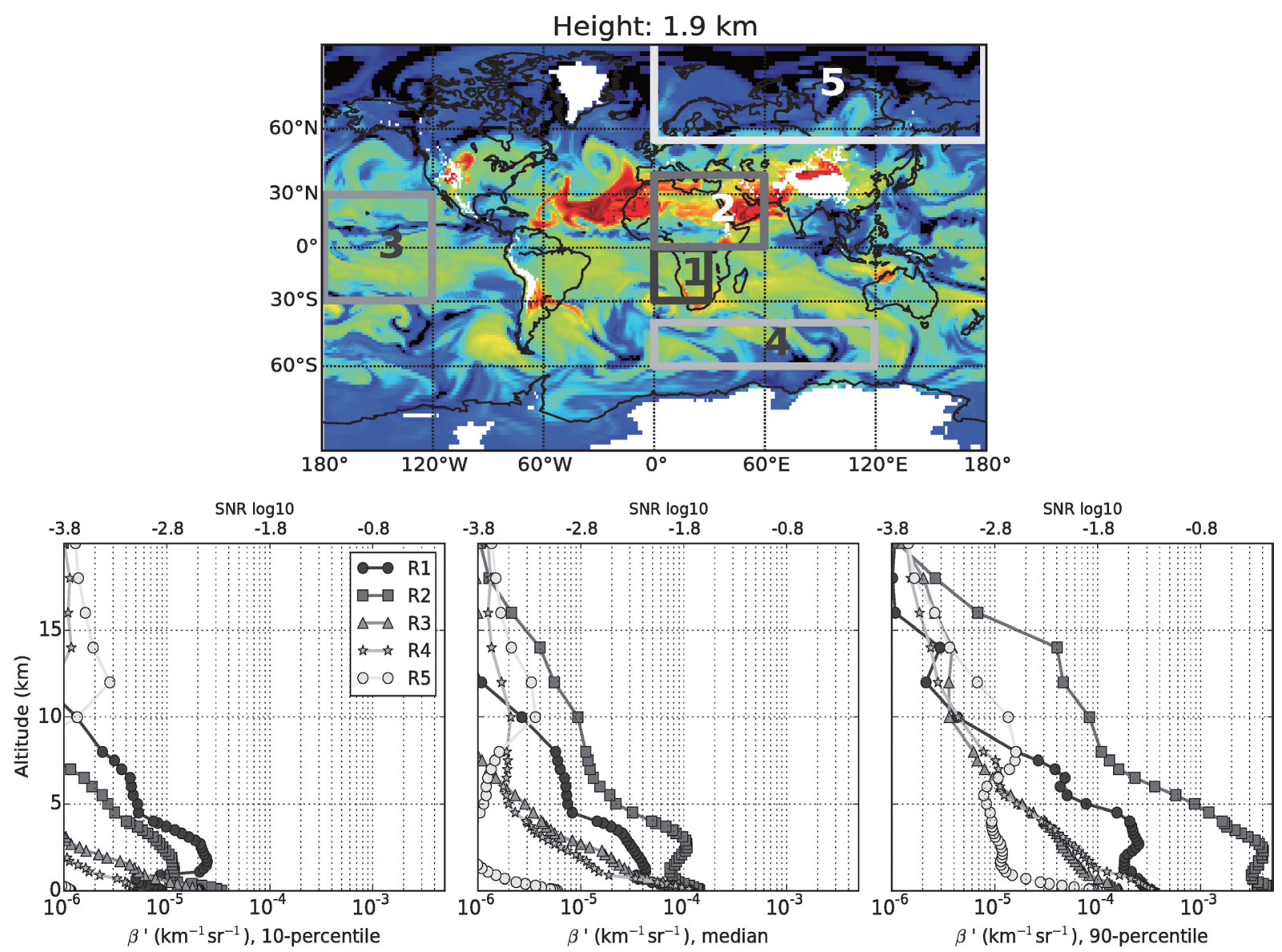

Fig. 3. Spatial variability of the aerosol attenuated backscatter coefficients at $2.05 \mu \mathrm{m}$. The upper panel shows the horizontal distribution of the coefficients at a height of $1.9 \mathrm{~km}$ with the same color scale as that in Fig. 2. The lower row shows, from left to right, 10, 50 and 90 percentile profiles of the coefficients within 5 selected regions indicated in the upper-panel. The upper-axis scale is the corresponding single-shot SNR estimated with Eq. (5) for a satellite altitude of $200 \mathrm{~km}$.

uously connected under the assumption that ice and liquid phases are equiprobable at $-25^{\circ} \mathrm{C}$. The backscatter and extinction coefficients are derived from the OPAC model (Hess et al. 1998) considering maritime and continental cases for stratus and cumulus liquid clouds, and cirrus- 1 and cirrus- 3 for ice clouds. The median attenuated backscatter coefficients range from $1 \mathrm{~km}^{-1} \mathrm{sr}^{-1}$ in the lower atmosphere down to $10^{-4}$ $\mathrm{km}^{-1} \mathrm{sr}^{-1}$ in the upper troposphere (Fig. 4).

\section{Signal computation and wind retrieval}

\subsection{Narrow-range signal}

A signal over a range gate $r$ with constant SNR and wind, is expressed as the following discrete time series (Zrnić 1979; Rye and Hardesty 1993; Frehlich and Yadlowsky 1994):

$$
z_{r, i}=\Xi_{i}+\sqrt{2}\left(\mathrm{SNR}_{r}\right)^{\frac{1}{2}} \Lambda_{i} \cos \left(2 \pi i \frac{F_{d, r}}{F_{s}}+\phi_{i}\right)
$$

with $i$ as the time index. The signal intensity $z_{r, i}$ is a real-valued number, $F_{d, r}$ is the Doppler frequency inside the gate $r, \mathrm{SNR}_{r}$ is the backscattered signal power and $\Xi$ is a Gaussian white noise such as $\left\langle\Xi_{i}^{2}\right\rangle=1$ (Eq. 4). The parameters $\Lambda_{i}$ and $\phi_{i}$ are independent zero-mean random parameters with exponential (variance $=1$ ) and uniform $(0-2 \pi)$ distributions, respectively. They both have auto-correlations with 1/e FWHM of $480 \mathrm{~ns}$ (72 m).

The theoretical power spectrum $(\mathrm{W} / \mathrm{Hz})$ of $z_{r}$ at a frequency $F_{k}$ is given by: 

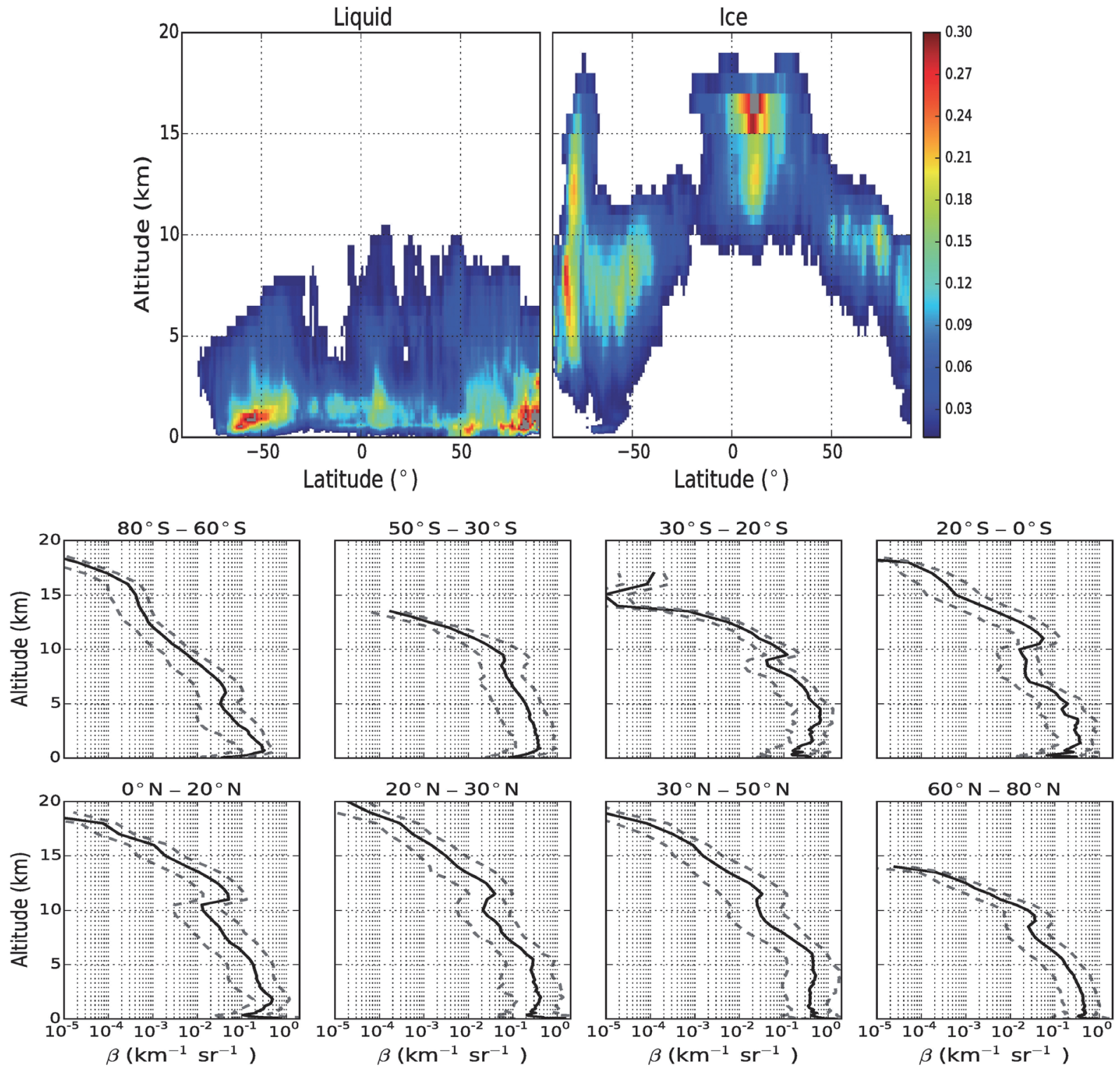

Fig. 4. Zonal distribution of the cloud backscatter coefficients at $2.05 \mu \mathrm{m}$. The upper panels show the zonal and altitude distribution of the cloud coverage ratio for liquid (left panel) and ice (right panel) clouds. The lower panels show the zonal median profiles of the backscatter coefficient (full lines) in various latitude bins. The dashed lines show the 10 and 90 percentile profiles.

$$
\mathrm{P}_{r}\left(k, F_{d, r}\right)=\frac{\left\langle\Xi_{i}^{2}\right\rangle}{F_{s}}+\frac{\mathrm{SNR}_{r} / 2}{\sqrt{2 \pi} w_{r}}\left[g\left(F_{k}+F_{d, r}, w_{r}\right)+g\left(F_{k}-F_{d, r}, w_{r}\right)\right],
$$

where $g$ is a Gaussian function with a standard-deviation width $w_{r}$ and $F_{k}=k F_{s} / M(k=0, \ldots, M / 2-1)$.

The signal $z_{r}$ is generated using the fast algorithm described in (Zrnić 1979; Frehlich and Yadlowsky
1994). The computation of an M-samples time series is performed in three steps. (1) The theoretical power spectrum $\mathrm{P}_{r}(k)$ (Eq. 8) is calculated with a spectral resolution $F_{s} / M^{\prime}$ with $M^{\prime}=2 M$. (2) $M^{\prime}$ random com- 
plex-valued Fourier coefficients $\epsilon_{k}$ are created considering a zero-mean Gaussian distribution and variances such as $\left\langle\operatorname{Re}\left(\epsilon_{k}\right)^{2}\right\rangle=\left\langle\operatorname{Im}\left(\epsilon_{k}\right)^{2}\right\rangle=M^{\prime} F_{s} P_{r}(k) / 2(\operatorname{Re}$ and Im denote the real and imaginary components). The real and imaginary coefficients are independent, the positive frequency coefficients are mutually independent, and the negative and positive frequency coefficients are related by the symmetrical rules for realvalued signals: $\operatorname{Im}\left(\epsilon_{k}\right)=-\operatorname{Im}\left(\epsilon_{M^{\prime}-k}\right)$ and $\operatorname{Re}\left(\epsilon_{k}\right)=\operatorname{Re}$ $\left(\epsilon_{M^{\prime}-k}\right)$. (3) Finally, the inverse Fourier transform of $\epsilon$ gives a time-domain signal ( $M^{\prime}$ samples) from which $M$ consecutive samples are extracted to obtain $z_{r}$.

Effects of wind variabilities over the narrow range gates $\Delta_{r}$ are partly included as a broadening of the theoretical narrow-range spectrum (Frehlich 2000). The width $\omega_{r}$ (Eq. 8) is:

$$
\omega_{r}^{2}=\omega_{p}^{2}+\omega_{a t m, r}^{2}+\omega_{L O}^{2},
$$

where $\omega_{p}=0.937 \mathrm{MHz}$ is the standard deviation (STD) width induced by the laser pulse, $\omega_{L O}$ is the local oscillator frequency uncertainty and $\omega_{a t m, r}$ is the effect of the wind variability over the range $r$. The parameter $\omega_{L O}$ has been estimated to $0.1 \mathrm{MHz}$ with ground-based observations (Iwai et al. 2013), and $\omega_{r ; \text { atm }}$ is parametrized as:

$$
\omega_{r, a t m}^{2}=0.01 \times F_{d, r}^{2}+\omega_{T}^{2},
$$

where $\omega_{T}=0.5 \mathrm{MHz}$ at altitudes below $3 \mathrm{~km}$ and 0 above. This includes effects from horizontal-wind vertical shears $\left(\omega_{\text {shear }}\right)$ and turbulences $\left(\omega_{\text {tur }}\right)$. Typically, large shear $H_{s}=20 \mathrm{~m} \mathrm{~s}^{-1} \mathrm{~km}^{-1}$ is found in the boundary layer and in jet streams (Salamitou et al. 1995; Frehlich 2000), which corresponds to

$$
\omega_{\text {shear }}=H_{s} \frac{\Delta_{r} \sin (\theta) \cos (\theta)}{2 \sqrt{12}}=0.27 \mathrm{~m} \mathrm{~s}^{-1},
$$

for a Nadir angle of $\theta=35^{\circ}$ and $\Delta_{r}=96 \mathrm{~m}$ along the LOS $(M=256)$. Strong turbulences are found in the boundary layer with typical values (Frehlich 1997; Wu et al. 2013):

$$
\omega_{\text {turb }}=0.671\left(\epsilon \Delta_{r}\right)^{1 / 3}=0.41 \mathrm{~m} \mathrm{~s}^{-1},
$$

where $\epsilon=0.0025 \mathrm{~m}^{2} \mathrm{~s}^{-3}$ is the energy dissipation factor. It is difficult to properly describe such processes in ISOSIM since the information for small scale turbulences is not available in the model. However, our simple parametrization (Eq. 10) covers the variability ranges of such processes and produces different effects in the lower and upper atmospheres and large values in the jet streams $(\approx 3 \mathrm{MHz})$. In the future, a global climatology for wind turbulence such as in (Frehlich and Sharman 2010) could be used to improve the model.

\subsection{From narrow-range to average spectra}

Narrow-range power spectra density is calculated as

$$
\hat{P}_{r, M}\left(k, F_{d, r}\right)=\frac{\left|\operatorname{FFT}\left(z_{r}\right)\right|_{k}^{2}}{M F_{s}},
$$

and averaged as

$$
\hat{P}_{M, N_{a}}(k)=\frac{1}{N_{a}} \sum_{r=1}^{N_{a}} \hat{P}_{r, M}\left(k, F_{d, r}\right) .
$$

For the retrieval spatial resolutions considered in this analysis $\left(50-200 \mathrm{~km}^{2}\right), N_{a}=1000-4000$ narrow-range spectra are averaged along the LOS and the orbit track. The narrow-range characteristics $\left(F_{d, r}, \omega_{r}\right.$ and $\mathrm{SNR}_{r}$ ) vary over the retrieval range. In particular, the cloudiness resolution $(10 \mathrm{~km}$ along the horizontal and $200 \mathrm{~m}$ along the vertical) produces random highly inhomogeneous 2-d field of SNR. Large scale inhomogeneities are then fully taken into account and bring additional wind retrieval uncertainties as shown in Section 5. Additional retrieval uncertainties arise from the spectrum broadening due to the signal truncation in the narrow-range gates. Also an inhomogeneous wind field modifies the line shape of the average-spectrum which is not a Gaussian function as described in Eq. (8).

\subsection{Frequency Retrieval}

The frequency retrieval is performed in two steps. First, the Doppler frequency is retrieved from the maximum of the log-likelihood function derived from the average spectrum $\hat{P}_{M, N_{a}}$ (Frehlich and Yadlowsky 1994; Rye and Hardesty 1997; Dabas et al. 2000). Thought it is possible to retrieve the spectral width and SNR simultaneously (see Appendices 1 and 2), here only the frequency is retrieved to reduce the simulation computation time. However it is important to note that the uncertainties on the non-retrieved parameters are taken into account. Their values are designed as "a-priori" to highlight that they are different than the truth and are not retrieved.

The likelihood function is calculated on a coarse frequency grid $F_{a}$ as follows: 


$$
L\left(F_{a}\right)=\sum_{k=0}^{M / 2-1} \frac{-\hat{P}_{M, N_{a}}(k)}{\mathrm{P}\left(k, F_{a}, \mathrm{SNR}_{a}, \omega_{a}\right)},
$$

where $\mathrm{P}$ is the Gaussian theoretical spectrum (Eq. 8), $\omega_{a}$ and $\mathrm{SNR}_{a}$ are the a-priori values of the width and SNR of the average spectrum. To reduce computational time, the grid $F_{a}$ is chosen to be the same as the coarse grid $F_{k}$ on which $\hat{P}_{M, N_{a}}$ is defined $(\Delta F=1.56$ $\mathrm{MHz}$ ).

In the second step, the frequency retrieval is improved using the 2nd-order polynomial fit of $L$ around its maximum:

$$
\widehat{F_{d}}=\Delta F\left[q_{\max }+\frac{L_{q_{\max }-1}-L_{q_{\max }+1}}{2\left(L_{q_{\max }-1}+L_{q_{\max }+1}-2 L_{q_{\max }}\right)}\right],
$$

where $q_{\max }$ is the frequency index of the likelihood maximum.

The spectral width a-priori $\omega_{a}$ is defined as:

$$
\omega_{a}=\sqrt{\bar{\omega}_{r}^{2}+\omega_{F_{d}, N_{a}}^{2}}+0.25
$$

where $\bar{\omega}_{r}$ is the average of $\omega_{r}$ (Eq. 9) over the $N_{a}$ narrow-ranges:

$$
\bar{\omega}_{r}=\frac{1}{N_{a}} \sum_{r=1}^{N_{a}} \omega_{r}
$$

and $\omega_{F_{d, N} N_{a}}$ is the standard deviation of $F_{d, r}$ :

$$
\omega_{F_{d}, N_{a}}^{2}=\overline{F_{d, r}^{2}}-\left(\bar{F}_{d, r}\right)^{2} .
$$

Throughout the manuscript, the upper-bar - refers to the average operation defined in Eq. (15). Because of the variability of the narrow-range SNR (speckle noise and spatial inhomogeneities), such an average operation is only an approximation of the true value of the average-spectrum parameter. The additional term $0.25 \mathrm{MHz}$ in Eq. (14) is to account for the effects of the signal truncation in the narrow range gates. The broadening of the average spectrum varies between $0.2-0.5 \mathrm{MHz}$ depending on signal characteristics (Fig. 13 in Appendix 1) and Eq. (14) can then be considered as an approximation with a random error of $0.1-0.15$ $\mathrm{MHz}$.

A fixed value of the SNR a-priori is used:

$$
\mathrm{SNR}_{a}=0.1 .
$$

It will be shown here that this rough assumption does not degrade the frequency retrievals but, on the contrary, it helps to mitigate a frequency retrieval bias for spectra with SNR $>0.1$ which is likely due to the time-truncation induced line-shape broadening.

Figure 5 shows the standard deviations (STDs) and the biases for the retrieval errors $\left(\widehat{F_{d}}-F_{d}\right)$ calculated from an ensemble of signals with SNR $=0.01$ (upper row) and SNR $=10$ (lower row). Here we consider an ideal case in order to assess the retrieval algorithm performance. The signal parameters (Doppler frequency, spectrum width, SNR) are constant over the $N_{a}$ averaged ranges $\left(F_{d, r}=F_{d}\right.$ and $\left.\omega_{F_{d}, N_{a}}=0\right)$. The Doppler frequency $F_{d}$ is randomly changed from one retrieval to another such that the range $100 \pm \Delta_{F} / 2 \mathrm{MHz}$ is uniformly covered. The a-priori spectral width corresponds to the mean value retrieved from the signal (see Figure legends). If the true SNR value is used for $\mathrm{SNR}_{a}$, the retrievals are biased by $0.05-0.1 \mathrm{MHz}$ when the true frequency $F_{d}$ is a fraction of $\Delta_{F}$ (first column panels). The STD is consistent with the Cramer-Rao lower bound (CRLB) which represents the best theoretical precision (see Appendix 1), and it is correlated with the derivative of the biases as expected from the theory (Eq. 36 in Appendix 1). Using $\mathrm{SNR}_{a}=0.1$ instead of the true value significantly reduces the retrieval biases but with a small cost on the standard deviation which becomes 10-20\% larger than the CRLB (second column panels).

A systematic analysis has been performed to assess the retrieval algorithm performances using the same experimental setting and $S N R_{a}=0.1$. Signals are generated with $\omega_{r}$ equals to 0.937 and $3 \mathrm{MHz}$. Retrievals with a probability larger than $50 \%$ to be "bad retrievals" (see Section 4.4) and those with an error $\left|F_{d}-F_{d}\right|>10 \mathrm{MHz}$ are rejected. The retrieval precision is defined as follows:

$$
e_{p, 10}=\operatorname{STD}\left(\widehat{F_{d}}-F_{d}\right) .
$$

For SNR values smaller than 1, the error STD follows the CRLB (Fig. 6) and, consistent with the previous discussion, it is a little larger than the CRLB for SNR $<10^{-2}$. The errors increase with the spectral width as $\omega^{1.5}$ (not shown). For SNR $>1$ the retrieval precision tends to remain constant when the SNR increases while the CRLB decreases. These are consistent results with those obtained from ground-based DWL measurements (Iwai et al. 2013). For large $N_{a}$ and SNR, a precision as low as $10^{-2} \mathrm{MHz}$ is obtained which demonstrates that the errors induced by the retrieval algorithm are negligible. 

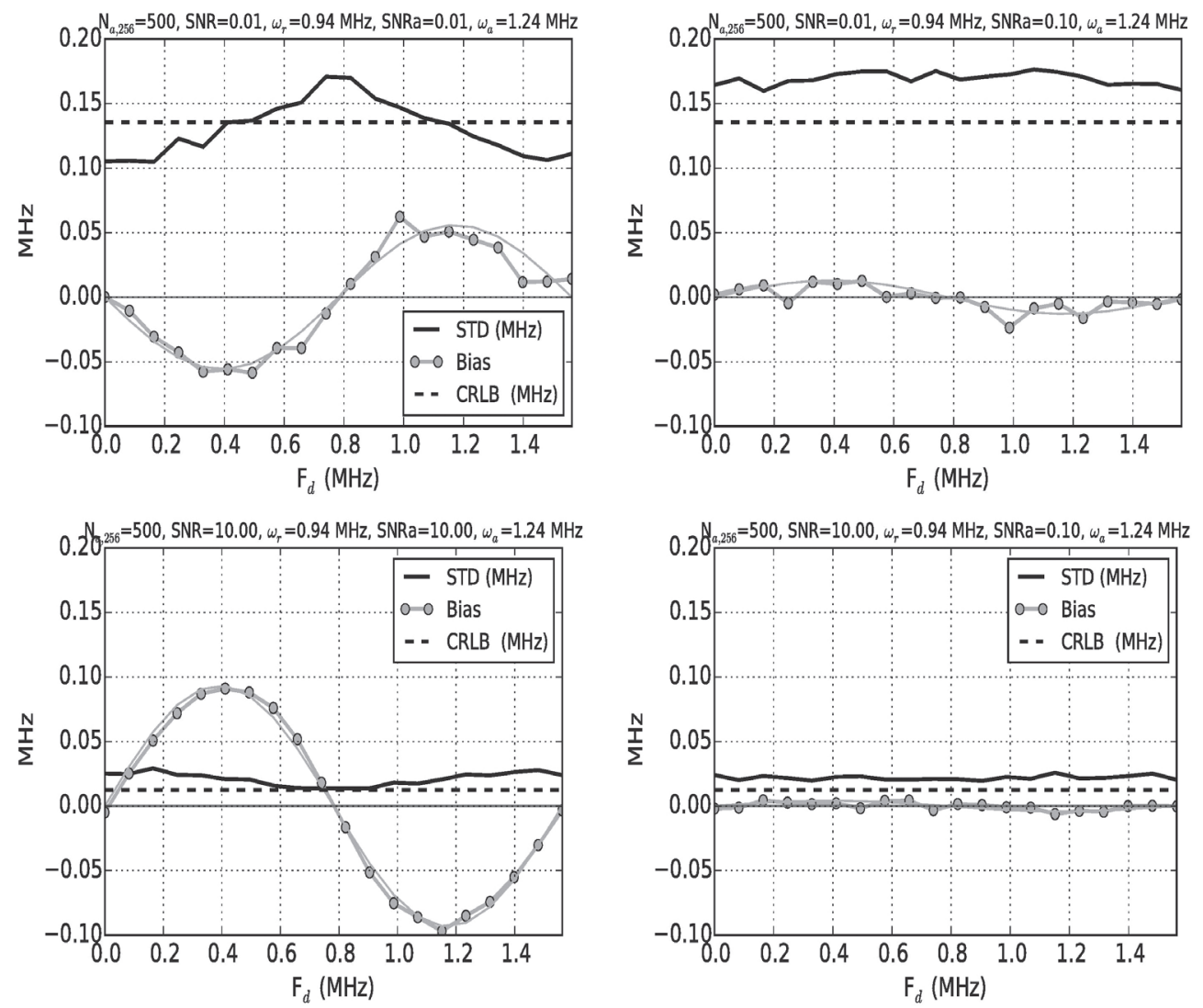

Fig. 5. Frequency retrieval errors as a function of the position of the Doppler frequency with respect to two adjacent spectrum frequencies $\left(\Delta_{F}=1.56 \mathrm{MHz}\right)$. The first row shows results for a signal with a narrow-range $\mathrm{SNR}=0.01$ and spectral width $w_{r}=0.94 \mathrm{MHz}$, while in the second row, a signal with narrow-range SNR $=10$ is used. The first column shows the results for an SNR a priori equal to that of the signal. The second column shows the results for the SNR a priori set to 0.1. The full black line is the STD of the retrieval errors, the dashed line is the unbiased CRLB (Appendix 2, Eq. 27) calculated for the a priori spectral width (Eq. 14), and the clear-gray squared lines show the retrieval biases. The thin full lines are the bias fits using a sin function.

\subsection{Bad and good retrievals}

An ensemble of frequency retrievals is composed of bad retrievals uniformly distributed over the spectral bandwidth $B$, and good retrievals with a Gaussian probability function centered at the true Doppler frequency $F_{d}$ (Frehlich and Yadlowsky 1994). Considering that all good retrievals are inside a range $\Delta_{b}$, the ratio of bad retrievals $r_{b}$ is:

$$
r_{b}=\frac{N_{b} / N_{w}}{\left(B-2 \Delta_{b}\right) / B},
$$

with $N_{w}$ is the total number of retrievals and $N_{b}$ is the number of retrievals with values outside the range $F_{d}$ $\pm \Delta_{b} \mathrm{MHz}$. We use $\Delta_{b}=20 \mathrm{MHz}$ such that $\Delta_{b} \gg e_{p, 10}$.
For unbiased retrievals, the general expression $e_{p, \Delta}$ of the retrieval precision defined in Eq. (18) is:

$$
e_{p, \Delta}^{2}=\frac{r_{b} \Delta^{3}}{12 B}+\left(1-r_{b}\right) \sigma_{g}^{2}
$$

where $\sigma_{g}$ is the standard deviation of the good retrievals. In Section 4.3, we have chosen $\Delta=10 \mathrm{MHz}$, and for $r_{b} \approx 0.1$, the bad-retrieval term in Eq. (20) brings an error of $0.2 \mathrm{MHz}$ while $e_{p, 10}$ is typically $0.5 \mathrm{MHz}$ (not shown). Given that $r_{b}$ quickly vanishes for increasing SNR (Frehlich and Yadlowsky 1994), we can consider that for $r_{b}<0.1, \Delta=10 \mathrm{MHz}$ is large enough to account for all good-retrievals $\left(\sigma_{g}<0.5 \mathrm{MHz}\right)$, and the impacts of bad-retrievals are not significant. This 


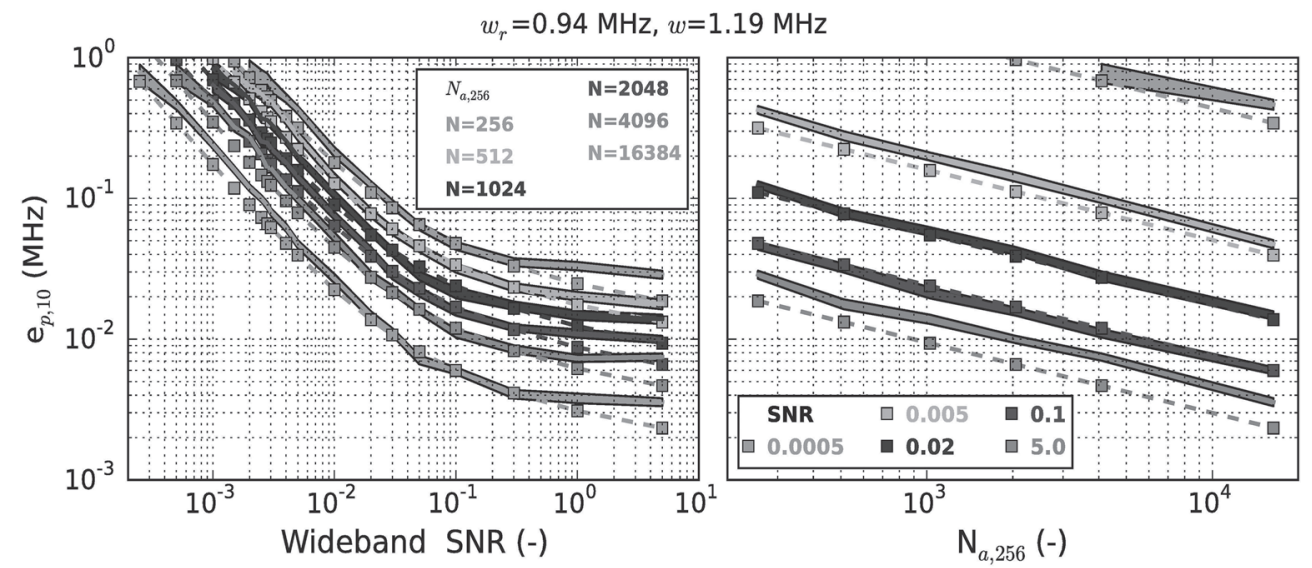

$w_{r}=3.00 \mathrm{MHz}, w=3.20 \mathrm{MHz}$
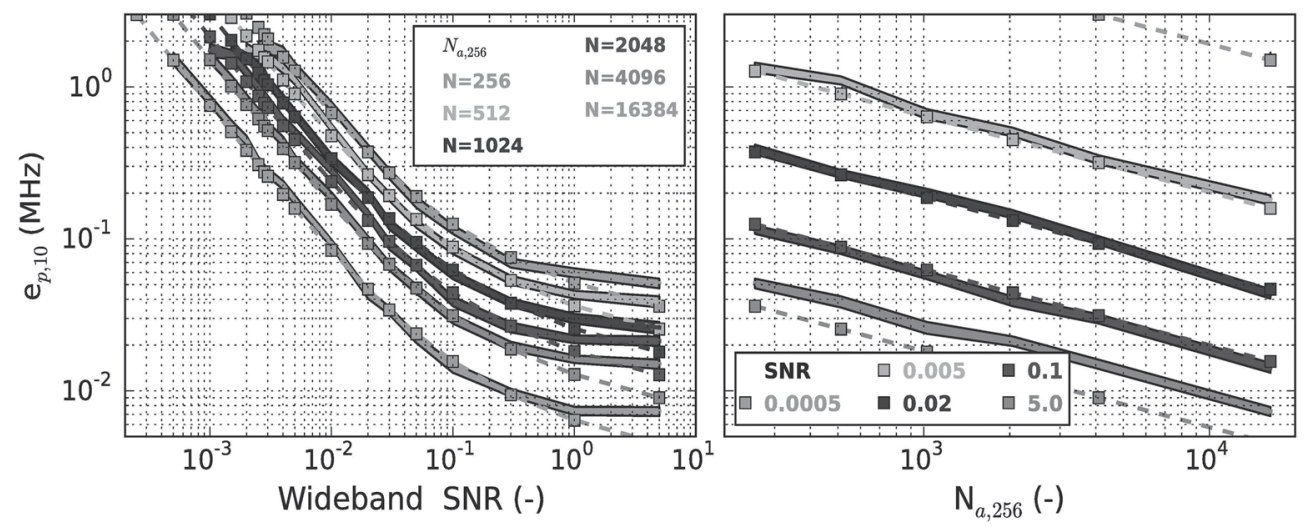

Fig. 6. Retrieval error estimation for different numbers of averaged narrow-range gates $\left(N_{a, 256}\right)$ and narrow-range SNRs. The upper and lower rows show the results for signal spectral widths of $w_{r}=0.94$ and $3 \mathrm{MHz}$, respectively. The a priori spectral width $w$ is $1.19 \mathrm{MHz}(3.20 \mathrm{MHz})$ for $w_{r}=0.94 \mathrm{MHz}(3 \mathrm{MHz})$. The a priori SNR is fixed to 0.1 for all cases. The dashed lines show the CRLB (Appendix 2, Eq. 27) computed for the apriori spectral width (Eq. 14).

shows that $r_{b}<0.1$ is a safe criterion for selecting good retrievals and $e_{p, 10}$ is a good approximation of the retrieval errors.

\section{Results from ISOSIM}

\subsection{Simulation setting}

A simulation of a full month period starting on August 1st 2010 is analyzed in this section. The satellite is on a sun-synchronous near-polar orbit with an inclination of $96.37^{\circ}$ and the local time of the ascending node is 7:30 am. The orbit Two-Line-Element (TLE) is given in Table 4. The satellite altitude varies from $218 \mathrm{~km}$ at the Equator to $228 \mathrm{~km}$ at the highest latitudes $\left(85^{\circ}\right)$. Only data from one of the two detectors are considered and the pulse shot rate is $15 \mathrm{~Hz}$ $(\mathrm{PRF} / 2)$. The retrieval layers are defined with respect to the Earth surface. The first retrieval layer has a vertical thickness of about $100 \mathrm{~m}$ and contains samples contaminated by the surface reflections. Samples from this layer are not included in the study. The second retrieval layer contains 5 narrow-ranges with center altitudes between 100 and $500 \mathrm{~m}$ (vertical resolution of $5 \times 78.6 \mathrm{~m}=393 \mathrm{~m}$ ). Similarly, a whole number of narrow-ranges is averaged so that the retrieval layer resolutions are close to $0.5,1$ and $2 \mathrm{~km}$ between 0.5 and $3 \mathrm{~km}, 3$ and $8 \mathrm{~km}$, and above $8 \mathrm{~km}$, respectively. The upper retrieval boundary is set to $20 \mathrm{~km}$. The retrieval horizontal resolution is $100 \mathrm{~km}$ along the orbit track (195 shots per detector).

The retrieval error (Eq. 18) is computed as follows:

$$
e_{p, 10}=\operatorname{STD}\left(\widehat{F_{d}}-\overline{F_{d}}\right),
$$


Table 4. Two Line Element (TLE) for the near-polar orbiting satellite considered in Section 5. Those lines are a standard format to compute the orbit parameters using suitable software available on the web. The first line contains the reference time (year 2010 and day 213.00) and in the second line, information about the orbit inclination $\left(96.36^{\circ}\right)$, the right ascension of the ascending node $\left(39.54^{\circ}\right)$ and the number of revolutions per day (16.23) are easily seen.

1 99999U XXXXXXXX 10213.00000000.00000000 00000-0 00000-0 000005 299999096.3683039 .54040015195329 .6858315 .245716 .23272445000013

where the STD is calculated over a subset of ISOSIM retrievals, $\widehat{F}_{d}$ is one of the retrieved frequency and $\overline{F_{d}}$ is the average of the narrow-range frequencies:

$$
\overline{F_{d}}=\frac{1}{N_{a}} \sum_{r=1}^{N_{a}} F_{d, r} .
$$

Figure 7 shows the zonal median of the LOS wind standard deviations $\left(\omega_{F_{d}, N_{a}}\right.$ in Eq. 16). A correlation between the thickness of the retrieval layers and $\omega_{F_{d} N_{a}}$ is noticeable. The values of the latter are typically $<$ $0.5 \mathrm{MHz}$ near the surface, $0.5-1 \mathrm{MHz}$ between 3 and $8 \mathrm{~km}$, and between 1 and $3 \mathrm{MHz}$ above $8 \mathrm{~km}$. This in- dicates that the wind inhomogeneities over the vertical direction dominate those in the horizontal direction. However it has to be kept in mind that the wind inhomogeneities over small horizontal scales $(<100 \mathrm{~km})$ are underestimated because of the coarse resolution of the atmospheric model.

Figure 8 shows a simulation of $40 \mathrm{mn}$ observation when the satellite moves from the South pole to the North pole. Above $5 \mathrm{~km}$, the average SNR values $(\overline{\mathrm{SNR}})$ exhibit variations of several orders of magnitude (left main panel) due to cloud induced signal enhancement much larger than the aerosol signal background. The average frequencies $\left(\overline{F_{d}}\right)$ and their
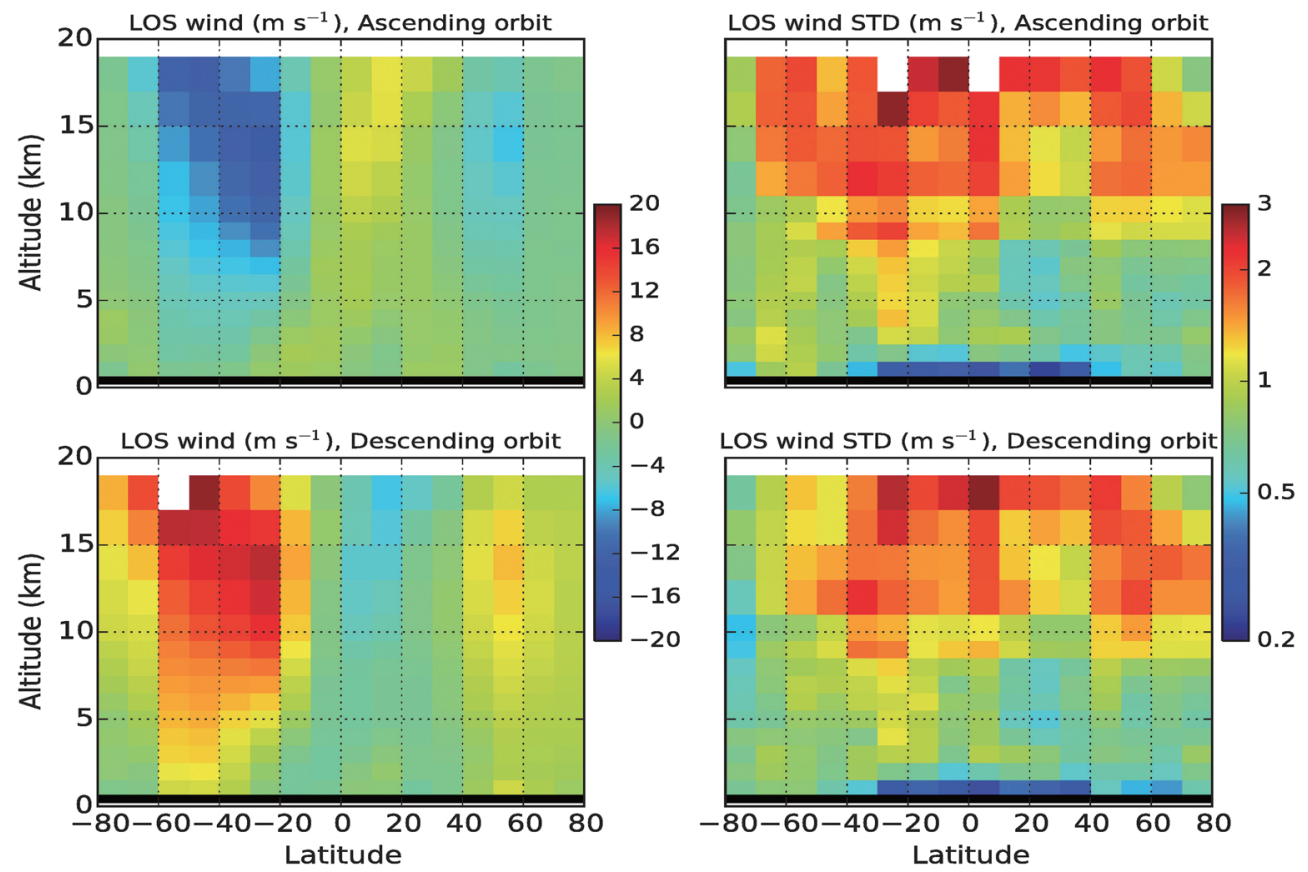

Fig. 7. Median of the zonal distributions of the LOS wind profiles (left panels) and of the narrow-range LOS wind standard-deviation $\left(\sim \omega_{F_{d}, N_{a}}\right)$ (right panels). The first (second) row shows results for the ascending (descending) branch where the instrument points northward-eastward (southward-westward) with an azimuth angle of $45^{\circ}$. 

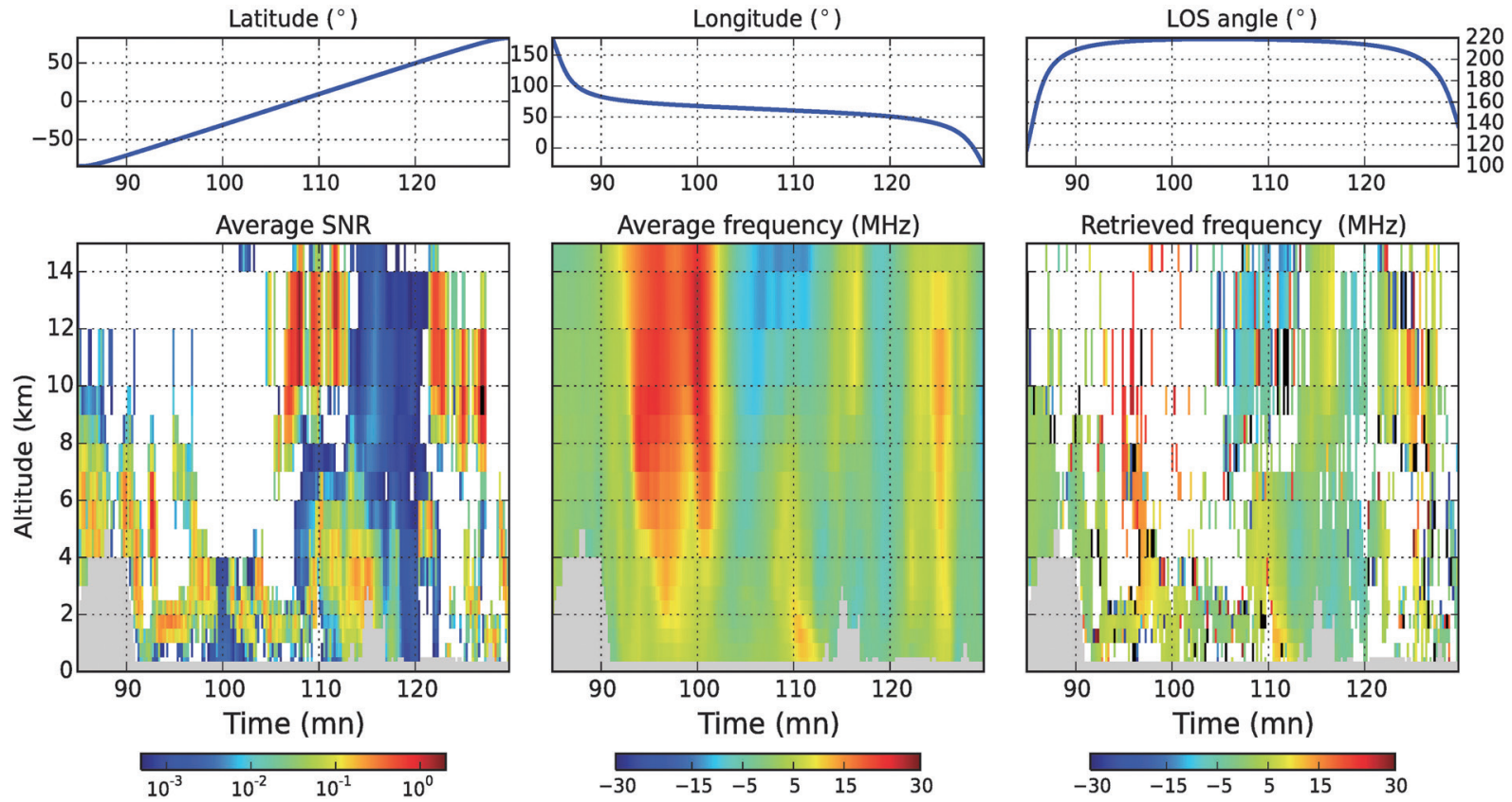

Fig. 8. Simulation of $40 \mathrm{mn}$ observation along the orbit track. The upper panels show from left to right the latitudes and the longitudes of the measurements, and the LOS orientation with respect to the North. The lower panels show the average narrow-range SNR $(\overline{\mathrm{SNR}})$ profiles, the average Doppler frequencies $\left(\overline{F_{d}}\right)$ (middle panel) and their "good" retrievals $\left(\widehat{F_{d}}\right)$. The same color scale is used for Doppler frequencies in the two last panels. The white area in the left-most panel is the range with $\overline{\mathrm{SNR}}<0.5 \times 10^{-3}$ The white and black areas in the right-most panel indicate regions with low probability for good retrievals $\left(\overline{\mathrm{SNR}} \sqrt{N_{a}}<0.08\right)$ and data with $\left|\widehat{F_{d}}-F_{d}\right|>5 \mathrm{MHz}$, respectively. Altitudes underneath $100 \mathrm{~m}$ above the surface are masked with gray color. The returns from these altitudes are contaminated by the surface.

retrieved values $\left(\widehat{F_{d}}\right)$ are shown in the second and third panels, respectively. Only the good retrievals are shown. In the middle and upper atmosphere the occurrence of good retrievals is clearly correlated to the presence of clouds or to the enhanced aerosol concentration in the northern mid-latitudes. Below $1 \mathrm{~km}$, areas with no good retrievals are frequent, likely because of signal absorption by clouds at higher altitudes.

\subsection{Good retrieval statistics}

The percentages of bad frequency retrievals in the ISOSIM dataset are shown in the lower panel of Fig. 9. Data with $\overline{\mathrm{SNR}} \times \sqrt{N_{a}}$ between 0.06 and 0.1 have a probability between $1 \%$ and $10 \%$ to be bad retrievals, independent of the altitude range. We refer data with a high probability for being good frequency estimates as "good retrievals", and consider that good retrievals are characterized by $\widehat{\mathrm{SNR}} \times \sqrt{N_{a}}>0.08$ where $\widehat{\mathrm{SNR}}$ is an estimate of $\overline{\mathrm{SNR}}$. This value has been chosen taking into account that the estimate precision of $\widehat{\mathrm{SNR}} \times \sqrt{N_{a}}=0.08$ is about 0.02 (Fig. 14 in Appen$\operatorname{dix} 2$ ).

Figure 10 shows the percentages of good retrievals for different latitude and altitude ranges. Below $3 \mathrm{~km}$ (layers 1-6), the percentage is between 25-55\% with the highest ratio in the Tropics and sub-Tropics at altitudes between 1-2 km (layers 3-4) (lower panel). The ratio of good retrievals below $1 \mathrm{~km}(\approx 30 \%)$ is significantly smaller than that of the layers above though the highest concentration of aerosols should provide more favorable conditions. The lower ratio is likely due to signal absorption by clouds above $1 \mathrm{~km}$.

Above about $3 \mathrm{~km}$, most of the good retrievals are performed if the signal is enhanced by reflections on the cloud top surface and sub-surface. Between 3 and $8 \mathrm{~km}$, the ratio of good retrievals is $35-45 \%$ at all latitudes except in the southern-Tropics, a region with low amount of cloudiness. There, the ratio is less than $15 \%$. 

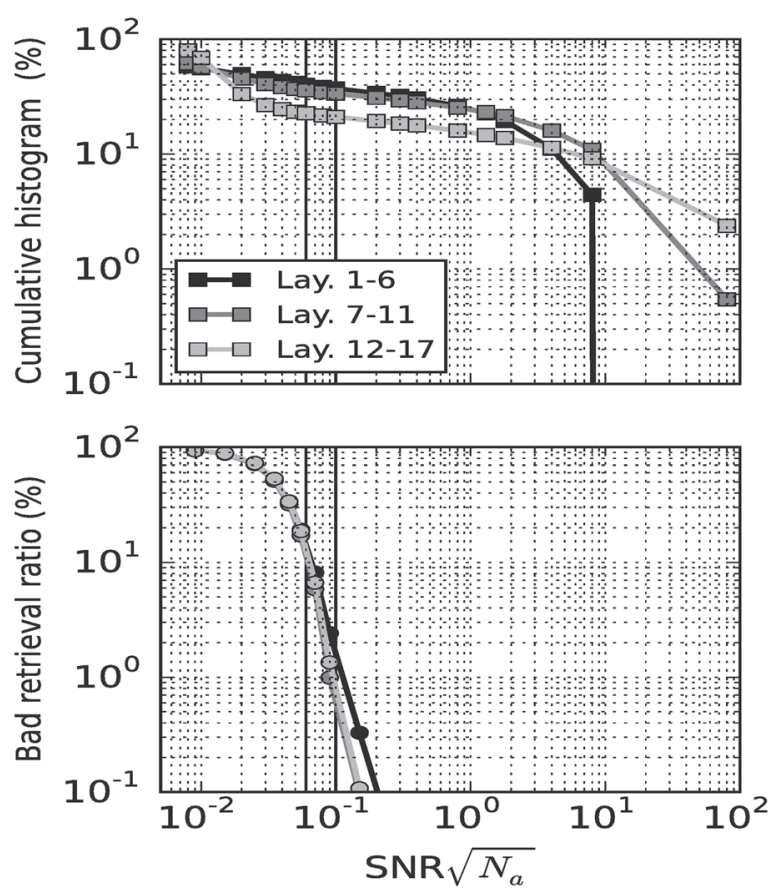

Fig. 9. Reverse cumulative distribution of $\overline{\mathrm{SNR}} \times$ $\sqrt{N_{a}}$ (upper panel) and bad frequency retrievals ratio with respect to $\overline{\mathrm{SNR}} \times \sqrt{N_{a}}$ (lower panel). The vertical lines indicate the range where $\overline{\mathrm{SNR}}$ $\times \sqrt{N_{a}}$ is between 0.06 and 0.1 . The SNR corresponds to that of narrow-range. Results are shown for retrieval layers $1-6,7-11$ and $12-$ 17 (see Table 1 for the corresponding altitudes).

For the upper-tropospheric layers $11-17$, the percentage of good retrievals is below $15 \%$, except in the northern Tropics and sub-Tropics where $25-45 \%$ of good retrievals are found. The relatively high level of good retrievals is correlated with the high ice-cloud occurrence rate in this region.

\subsection{Doppler frequency measurement performances}

The median of the retrieval errors (Eq. 21) is between $0.4-0.6 \mathrm{MHz}$ for the layers $1-11(<8 \mathrm{~km})$ while it increases to $0.8-1.2 \mathrm{MHz}$ for the layers $12-$ 17 (Fig. 11). In contrast to homogeneous cases, no clear correlation is found between the errors and $\overline{\mathrm{SNR}}$ $\times \sqrt{N_{a}}$ but, on the other hand, a correlation with the frequency standard-deviations $\omega_{F_{d}, N_{a}}$ (Eq. 16) is noticeable.

Figure 12 shows the errors with respect to $\omega_{F_{d, ~} N_{a}}$ for low values of $\overline{\mathrm{SNR}} \times \sqrt{N_{a}}$. For $\omega_{F_{d}, N_{a}}<0.2 \mathrm{MHz}$ and $\overline{\mathrm{SNR}} \times \sqrt{N_{a}}<0.25$, the measurement error is domi-
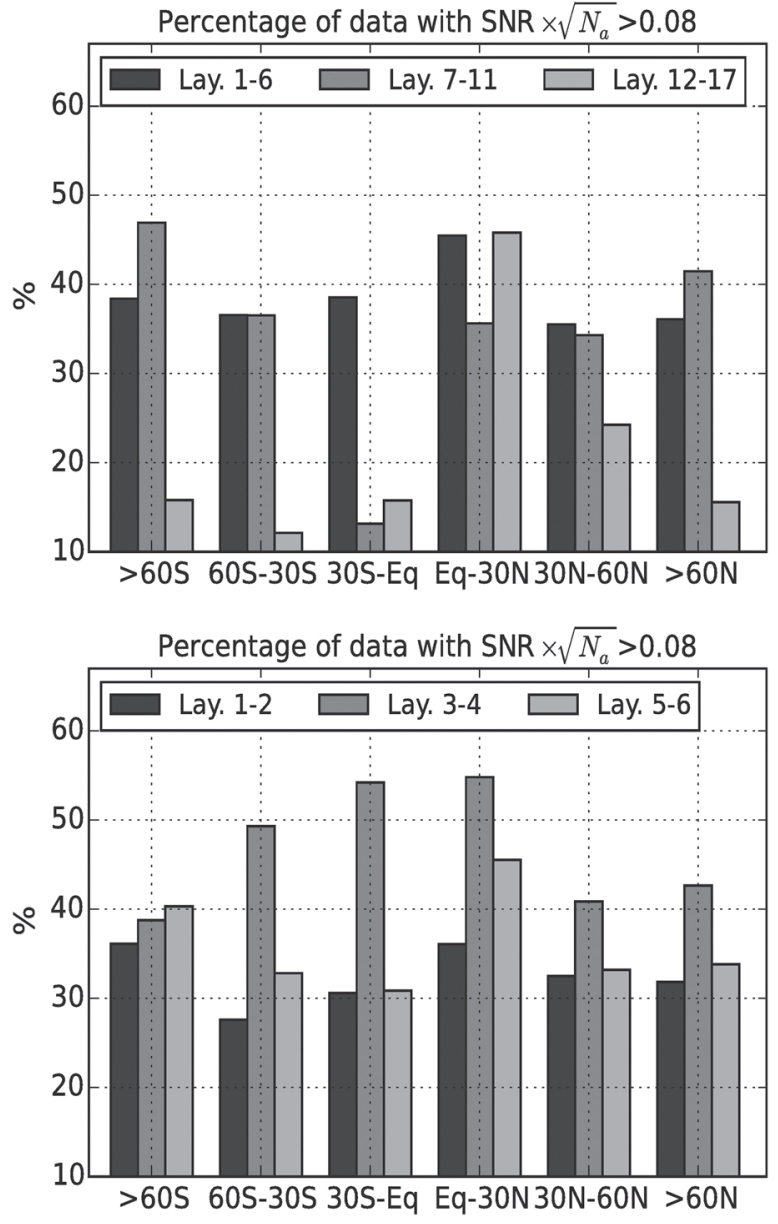

Fig. 10. Upper panel: Percentage of average spectra with $\overline{\mathrm{SNR}} \times \sqrt{N_{a}}$ larger than 0.08 for different latitude ranges and retrieval layers (SNR is that of a narrow-range gate). Results are shown for retrieval layers $1-6,7-11$ and 12-17 (see Table 1 for the corresponding altitudes). Lower panel: Same as the upper panel but for the lower troposphere: layers $1-2$ (altitude $<1 \mathrm{~km}), 3-4$ $(1-2 \mathrm{~km})$ and $5-6(2-3 \mathrm{~km})$.

nated by the measurement noise (detector and speckle noises) and fairly described by the CRLB. The highest turbulences over narrow ranges (included in $\omega_{T}$, Eq. 9) increase the retrieval errors in layers $1-6$ by about 50 $\%$ compared with higher altitudes with similar $\overline{\mathrm{SNR}} \times$ $\sqrt{N_{a}}$ and $\omega_{F_{d}, N_{a}}$.

For $\omega_{F_{d}, N_{a}}<0.2 \mathrm{MHz}$ and $\overline{\mathrm{SNR}} \times \sqrt{N_{a}}>0.4$, the measurement errors do not decrease below 0.1-0.2 $\mathrm{MHz}$ as expected from the CRLB. This is likely due to SNR variabilities. 

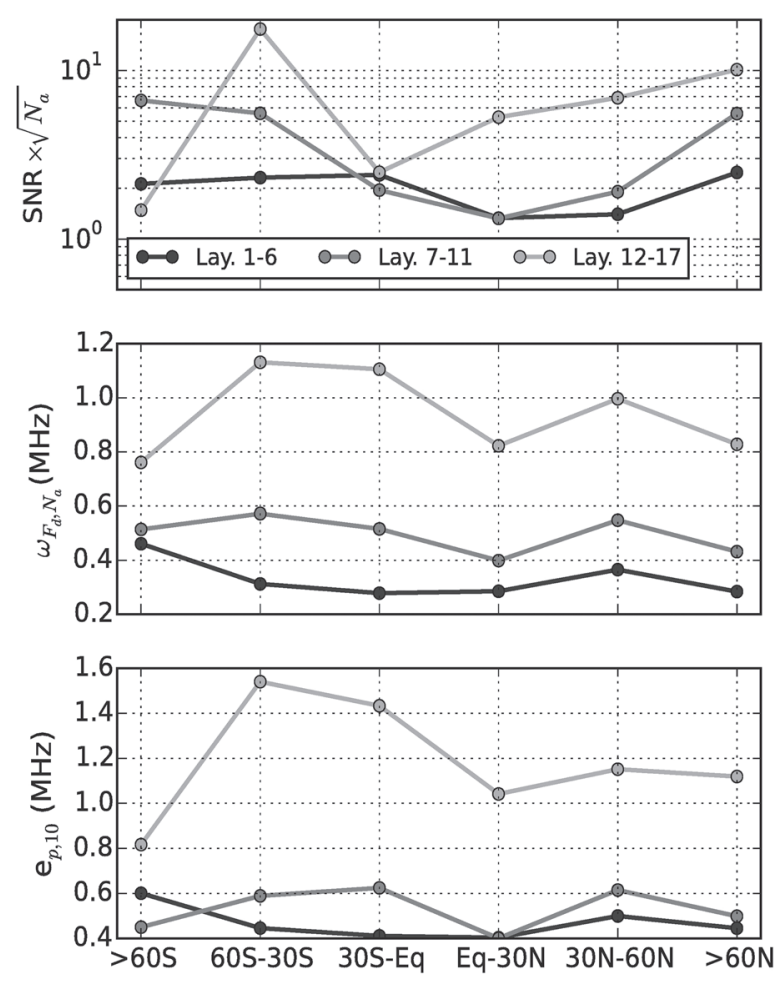

Fig. 11. Median of $\overline{\mathrm{SNR}} \times \sqrt{N_{a}}$ (upper panel), $\omega_{F_{d}, N_{a}}$ (middle panel) and $e_{p, 10}$ for the good retrievals $\left(\overline{\mathrm{SNR}} \times \sqrt{N_{a}}>0.08\right)$ with respect to latitude (SNR is that of a narrow-range gate). Results are shown for retrieval layers 1-6, 711 and $12-17$ (see Table 1 for the corresponding altitudes).

For $\overline{\mathrm{SNR}} \times \sqrt{N_{a}}>0.3$, the measurement error is correlated with $\omega_{F_{d}, N_{a}}$ for $\omega_{F_{d}, N_{a}}$ as low as $0.2 \mathrm{MHz}$. For smaller $\overline{\mathrm{SNR}} \times \sqrt{N_{a}}$, the correlation is clear for $\omega_{F_{d}, N_{a}}$ $>0.5 \mathrm{MHz}$.

The correlation of the measurement error with $\omega_{F_{d}, N_{a}}$ shows that, even with a perfect system, the measurement does not represent the mean velocity of an inhomogeneous scene. This error is similar to the representative error studied in (Frehlich 2000, 2001, 2004). Unlike those previous studies which considered different spatial samplings for the measurement and the true-wind definition, here the errors occur even though the samplings are the same (Eq. 22). However the true-wind calculation does not account for the weights of narrow ranges since the narrow-range SNR cannot be determined with enough precision (Appendix 2). This includes uncertainties in the truewind definition due to a combined effect of wind and
SNR variabilities, the SNR variability being mainly induced by clouds.

The aforementioned results were obtained with MASINGAR aerosol data v3. They are consistent with the results obtained from the previous version of MASINGAR (Ishii et al. 2016), though the aerosol backscatter coefficients derived from both versions show large differences (Section 3.2). The largest discrepancies are found for the ratio of mid/uppertropospheric good-retrievals at $30^{\circ} \mathrm{N}$, which are $20 \%$ smaller in the current study (MASINGAR-v3). The global consistency between the v2 and v3 results, let us think that in this analysis the cloudiness has a stronger impact on the measurement performances than the aerosol distribution.

\section{Conclusion}

A simulator for a feasibility study of a spaceborne infrared DWL has been presented. The results for a satellite orbiting on a near-polar orbit in summer time have been discussed in order to characterize the measurement performances with realistic conditions. In particular, the effects of atmospheric variabilities over the retrieval spatial resolution range are taken into account on the measured spectrum and on the true wind definition. Different a-priori and true parameters are used for the LOS wind retrievals. The dominant measurement error is induced by atmospheric variabilities over the observed range. This error source has to be taken into account for the OSSE to properly describe the differences between the pseudo-truth and the measurements.

The best measurement performances are found in the Tropics and southern mid-latitudes at altitudes between 1 and $2 \mathrm{~km}$ where more than $50 \%$ of the measurements provide relevant LOS wind information. Clouds are a key parameter for characterizing the measurement performances. The cloud top allows us to obtain most of the good retrievals in the mid and upper-troposphere (good retrieval ratio between $15 \%$ and $45 \%$ ), though the aerosol concentration is low. On the other hand, clouds shadow large area at low altitude. For instance, only $30 \%$ of good retrievals are found below $1 \mathrm{~km}$, even though it is where the highest aerosol concentration is found.

Simulated data for near-polar and low inclination orbits, for January and August have been created. They are being used in an OSSE to quantitatively assess the impacts of the mission on atmospheric models. These results are discussed in a companion paper (Okamoto et al. 2017).

Several improvements for the simulator have been 

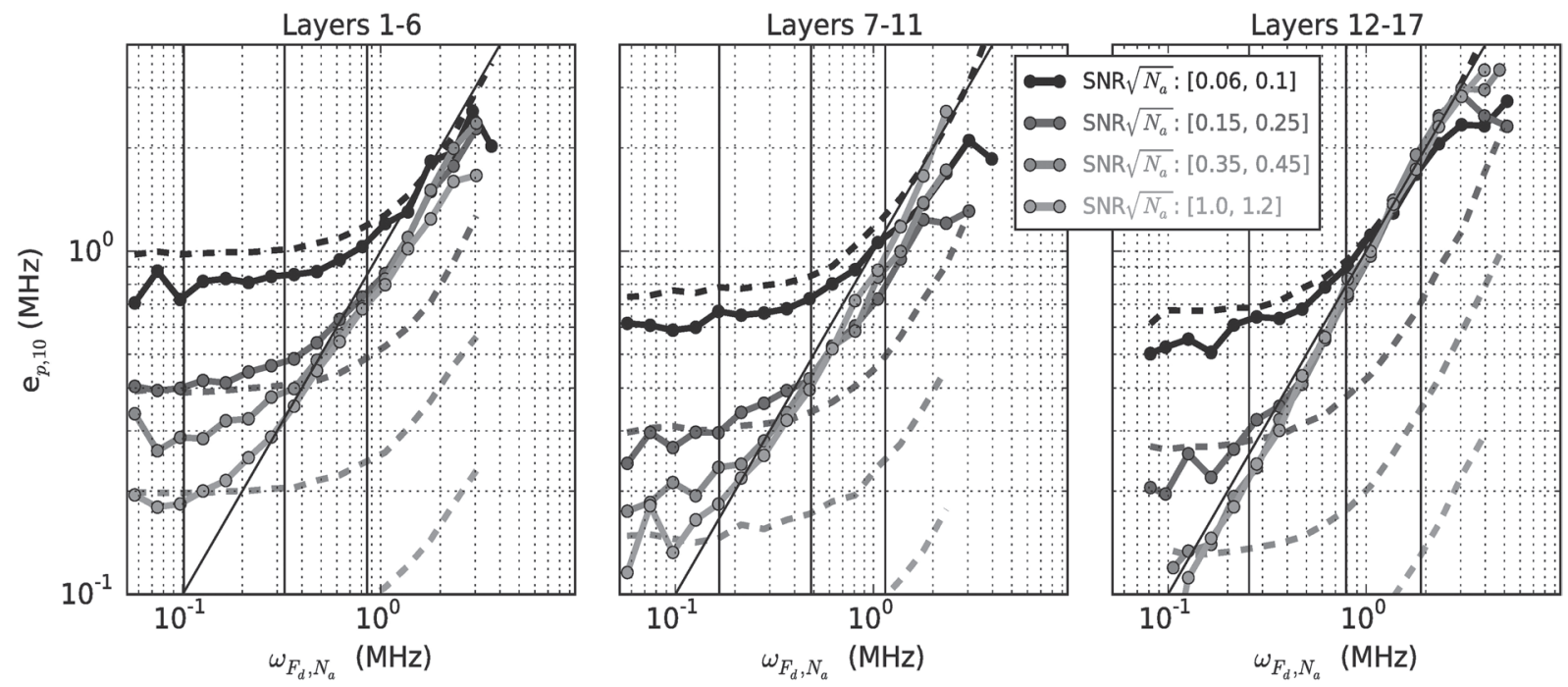

Fig. 12. Doppler frequency retrieval precision derived from the ISOSIM retrievals with respect to the narrowrange Doppler frequencies standard-deviation $\left(\omega_{F_{d}, N_{a}}\right)$. The dashed lines show the CRLB for homogeneous atmosphere and the vertical lines indicate the 10, 50 and 90 percentiles of the frequency standard-deviations. The slant line shows the " $y=x$ " line. The SNR is that of a narrow range gate. Results are shown for retrieval layers $1-6,7-11$ and $12-17$ (see Table 1 for the corresponding altitudes).

pointed out. A more suitable model is needed for simulating the surface returns which are used for studying pointing bias correction (not shown in this study). Aerosol backscatter coefficients should be calculated considering non-spherical particles. Atmospheric variabilities over small horizontal and vertical ranges should be improved but additional information on such variabilities is needed. For instance, the measurement performances at low altitudes depend on the probability for laser shots to propagate between clouds. This can only properly be estimated if the cloud spatial variability is described with a horizontal resolution better than the spacing between the laser shots $(<200 \mathrm{~m}$ for PRF $=30 \mathrm{~Hz})$. The model should also be validated with air-borne systems using similar wavelength.

\section{Appendix 1: Maximum likelihood estimator and Cramer-Rao lower bound}

The citations in the manuscript related to the Maximum Likelihood (ML) method deal with complexvalued signals. Here we derive the equations for the real-valued signals used in this study. The probability to measure a power $X_{k}$ in the spectral channel $k$ is described by a gamma distribution (Rye and Hardesty 1993):

$$
p_{N_{a}}\left(X_{k} \| \alpha\right)=\frac{\exp \left(-X_{k} / \chi_{k, N_{a}}(\alpha)\right)}{\left(N_{a}-1\right) ! \chi_{k, N_{a}}(\alpha)}\left(\frac{X_{k}}{\chi_{k, N_{a}}(\alpha)}\right)^{N_{a}-1}
$$

with

$$
N_{a} \chi_{k, N_{a}}(\alpha)=\left\langle X_{k}\right\rangle \approx \mathrm{P}_{k}(\alpha),
$$

and $N_{a}$ denotes the number of averaged independent spectra, $\mathrm{P}_{k}$ is the theoretical power spectrum (Eq. 8 ) and $\alpha$ is the signal parameters $\left(F_{d}, \omega, \mathrm{SNR}\right)$. The variance of $X_{k}$ is $\chi_{k, N_{a}}^{2}$, and if $N_{a}=1$, the distribution is exponential as expected from the Gaussian distribution of the Fourier coefficients of $X_{k}$. The logarithm of the likelihood function is:

$$
\begin{aligned}
\Lambda_{N_{a}}\left(X_{k}, \alpha\right) & =\ln \left(\prod_{k} p_{N_{a}}\left(X_{k} \| \alpha\right)\right) \\
& =K-N_{a} \sum_{k=0}^{M / 2-1}\left(\ln \mathrm{P}_{k}(\alpha)+\frac{X_{k}}{\mathrm{P}_{k}(\alpha)}\right)
\end{aligned}
$$

where $K$ is a parameter independent of $\alpha$ and $X$. It is shown using Bayesian theory that the signal parameters maximize the mean likelihood function: 

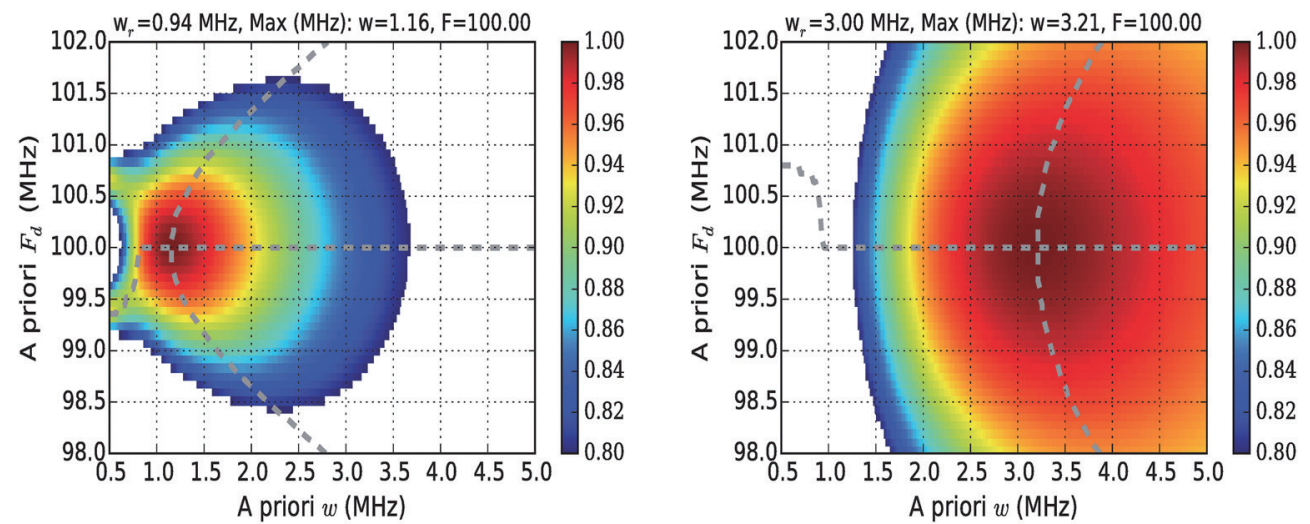

Fig. 13. Likelihood function with respect to the a priori Doppler frequency $\left(F_{d}\right)$ and spectrum width $(w)$. The function is derived from a set of simulated spectra with $w_{r}=0.937 \mathrm{MHz}$ (left panel) and $w_{r}=3 \mathrm{MHz}$ (right panel). Other parameters are $F_{d}=100 \mathrm{MHz}, \mathrm{SNR}=0.01$ and $N_{a}=100$. The vertical and horizontal dashed lines show the maximum location with respect to $w$ and $F_{d}$. The resolution of the likelihood function is 0.02 and 0.1 $\mathrm{MHz}$ for the $w$ and $F_{d}$ dimensions, respectively.

$$
\left\langle\frac{\partial \Lambda}{\partial \alpha}(\alpha)\right\rangle=0
$$

The average is performed over an ensemble of realizations of $X$. For a single realization, the maximum of $\Lambda$ is an estimate $\hat{\alpha}$ that is the most probable value of $\alpha$ given the measurement $X$.

Figure 13 illustrates the possibility to retrieve the Doppler frequency simultaneously with the spectral width. It shows the 2-D log-likelihood functions (Eq. $26)$ with respect to a priori spectral widths $\left(\omega_{a}\right)$ and Doppler frequencies $\left(F_{a}\right)$. The likelihood functions are derived from an ensemble of signals with $F_{d}=100$ MHz and $w_{r}=0.94 \mathrm{MHz}$ (left panel) and $w_{r}=3 \mathrm{MHz}$ (right panel). The horizontal lines show the values of the retrieved frequency (maximum of the likelihood) with respect to $\omega_{a}$. The retrieved frequencies correspond to the true frequency $F_{d}$ if $\omega_{a}$ is within 1-4 $\mathrm{MHz}$ which is the typical variability range of $\omega$ (Freh- lich 2000). The retrieved spectral widths are $\hat{\omega}=1.16$ $\mathrm{MHz}$ and $\hat{\omega}=3.21 \mathrm{MHz}$ (see text in each panel). They are larger than the correct values by $0.2-0.3 \mathrm{MHz}$ because of the spectral line broadening induced by the short observation time of the narrow range gates.

The Cramer-Rao lower bound (CRLB) defines the best theoretical retrieval precision. The CRLB $\sigma_{c r, \alpha}$ is defined as the lowest possible value of the variance of $\hat{\alpha}$. We follow the same steps used in (Rye and Hardesty 1993) for the complex valued signal but for considering the real-valued signal:

$$
\begin{aligned}
\sigma_{c r, \alpha}^{-2} & =\left\langle\left(\frac{\partial \Lambda}{\partial \alpha}\right)^{2}\right\rangle=-\left\langle\frac{\partial^{2} \Lambda}{\partial \alpha^{2}}\right\rangle \\
& =N_{a} \sum_{k=0}^{M / 2-1}\left(\frac{1}{\mathrm{P}_{k}} \frac{\partial \mathrm{P}_{k}}{\partial \alpha}\right)^{2},
\end{aligned}
$$

which gives:

$$
\begin{gathered}
\sigma_{c r, F_{d}}^{2}=\frac{1}{N_{a}}\left[\sum_{k=0}^{M / 2-1} \frac{\tilde{F}_{k}^{2}}{w^{4}}\left(1-\frac{1}{1+F_{s} \operatorname{snr} / 2 g\left(\tilde{F}_{k}, w\right)}\right)^{2}\right]^{-1}, \\
\sigma_{c r, \mathrm{snr}}^{2}=\frac{\operatorname{snr}^{2}}{N_{a}}\left[\sum_{k=0}^{M / 2-1}\left(1-\frac{1}{1+F_{s} \operatorname{snr} / 2 g\left(\tilde{F}_{k}, w\right)}\right)^{2}\right]^{-1}, \\
\sigma_{c r, w}^{2}=\frac{1}{N_{a}}\left[\sum_{k=0}^{M / 2-1}\left(\frac{\tilde{F}_{k}^{2}-w^{2}}{w^{3}}\right)^{2}\left(1-\frac{1}{1+F_{s} \operatorname{snr} / 2 g\left(\tilde{F}_{k}, w\right)}\right)^{2}\right]^{-1},
\end{gathered}
$$


with $\tilde{F}_{k}=F_{k}-F_{d}$ and $g\left(\tilde{F}_{k}, w\right)=\frac{1}{\sqrt{2 \pi} w} \exp \left(-\left(\tilde{F}_{k}^{2} /\left(2 w^{2}\right)\right)\right.$, and $\sigma_{c r, w}^{2}, \sigma_{c r, \text { snr }}^{2}$ and $\sigma_{c r, w}^{2}$ are the CRLB for the Doppler frequency, the SNR and the pulse width, respectively. For $\mathrm{SNR} \ll 1, w \ll F_{s}$ and using:

$$
\begin{aligned}
& \sum_{k=0}^{M / 2-1}\left(1-\frac{1}{1+F_{s} \operatorname{snr} / 2 g\left(\tilde{F}_{k}, w\right)}\right)^{2} \approx \frac{M F_{s} \mathrm{snr}^{2}}{8 \sqrt{\pi} w}=\mathrm{U}, \\
& \sum_{k=0}^{M / 2-1} \tilde{F}_{k}^{2}\left(1-\frac{1}{1+F_{s} \operatorname{snr} / 2 g\left(\tilde{F}_{k}, w\right)}\right)^{2} \approx \frac{w^{2}}{2} \mathrm{U}, \\
& \sum_{k=0}^{M / 2-1} \tilde{F}_{k}^{4}\left(1-\frac{1}{1+F_{s} \operatorname{snr} / 2 g\left(\tilde{F}_{k}, w\right)}\right)^{2} \approx \frac{3 w^{4}}{4} \mathrm{U},
\end{aligned}
$$

we obtain the following approximations:

$$
\begin{aligned}
& \sigma_{C R, F_{d}}^{2}(\operatorname{snr} \ll 1)=\frac{16 \sqrt{\pi} w^{3}}{M N_{a} F_{s} \operatorname{snr}^{2}}, \\
& \sigma_{C R, w}^{2}(\operatorname{snr} \ll 1)=\frac{32 \sqrt{\pi} w^{3}}{3 M N_{a} F_{s} \operatorname{snr}^{2}}, \\
& \sigma_{C R, \mathrm{snr}}^{2}(\operatorname{snr} \ll 1)=\frac{8 \sqrt{\pi} w}{M N_{a} F_{s}} .
\end{aligned}
$$

For biased estimates, the CRLB becomes (Rye and Hardesty 1993):

$$
\left(\sigma_{C R, \alpha}^{\prime}\right)^{2}=\left(1+\frac{d \varepsilon}{d \alpha}\right) \sigma_{C R, \alpha}^{2}
$$

where $\varepsilon=\langle\hat{\alpha}-\alpha\rangle$ is the estimator bias.

\section{Appendix 2: Simple SNR estimator}

The single-shot signal power $\overline{\mathrm{SNR}}$ can be estimated from the spectral integration of the average spectrum (Dabas et al. 1994; Bruneau et al. 2006). Since the backscattered line is narrow $(w=1-4 \mathrm{MHz})$ and its frequency is retrieved independently of the SNR with a relatively small error $(<2 \mathrm{MHz})$, integration can be performed over a small frequency range encompassing the backscattered line to reduce the measurement noise effects. The signal power estimate is then $\widehat{\mathrm{SNR}}$ $=\hat{p}-N^{\prime}$ with:

$$
\begin{aligned}
\hat{p} & =\frac{2 F S}{m N_{a}} \sum_{r=1}^{N_{a}} \sum_{k=k_{0}-m / 2+1}^{k_{0}+m / 2} \hat{P}_{k, M, r} \\
& =\frac{1}{2 m N_{a}} \sum_{r=1}^{N_{a}} \sum_{i=1}^{2 m} \tilde{z}_{r, i}^{2},
\end{aligned}
$$

where $k_{0}$ is the frequency index of the retrieved frequency, $m$ is the size of the reduced spectral range and $\tilde{z}$ is the corresponding time domain signal measured with a noise power of $N^{\prime}=2 \mathrm{~m} / \mathrm{M}$ and a sampling interval $T_{s}^{\prime}=M T_{s} / 2 \mathrm{~m}$. The variance of $\tilde{z}_{r, i}$ is

$$
\sigma_{\tilde{z}, r}^{2}=\left\langle\tilde{z}_{r, i}^{2}\right\rangle=N^{\prime}+\mathrm{SNR}_{r},
$$

where $\langle\bullet\rangle$ is the ensemble average. For a single homogeneous range gate $r$, the variance of $\hat{p}$ is (Rye and Hardesty 1997)

$$
\sigma_{p, r}^{2}=\frac{1}{m}\left(2 \sum_{0}^{2 m-1}(1-k / 2 m) R_{z z}^{2}(k)-\sigma_{\tilde{z}, r}^{4}\right),
$$

where $R_{\widetilde{z z}}$ is the autocorrelation function of $\tilde{z}$. This is derived noticing that the autocorrelation function of $y_{i}$ $=\tilde{z}_{i}^{2}$ is

$$
R_{\widetilde{\mathrm{zz}}}^{2}(i)=\frac{R_{\mathrm{yy}}(i)-R_{\mathrm{yy}}(\infty)}{2},
$$

with $\sigma_{y}^{2}=R_{\mathrm{yy}}(0)-R_{\mathrm{yy}}(\infty), R_{\mathrm{yy}}(\infty)=\sigma_{z}^{4}$ and for realvalued data, $R_{\mathrm{yy}}(0)=3 \sigma_{\tilde{z}}^{4}$. The variance of $\hat{p}_{r}$ (Eq. 39) is then derived in the following way:

$$
\begin{aligned}
\sigma_{p, r}^{2} & =\frac{1}{(2 m)^{2}}\left(\left\langle\sum_{i=0}^{2 m-1} y(i) \sum_{q=0}^{2 m-1} y(q)\right\rangle-\left\langle\sum_{i=0}^{2 m-1} y(i)\right\rangle^{2}\right) \\
& =\frac{1}{2 m}\left(2 \sum_{i=0}^{2 m-1} \Lambda(i) R_{y y}(i)-R_{y y}(0)-2 m \sigma_{z, r}^{4}\right) \\
& =\frac{1}{m}\left(2 \sum_{i=0}^{2 m-1} \Lambda(i) R_{z z}^{2}(i)-\sigma_{z, r}^{4}\right),
\end{aligned}
$$

where $\Lambda(i)=1-i / 2 m$. Using the following (Zrnić 1979)

$$
\begin{aligned}
R_{\widetilde{z z}}(k)= & N^{\prime} \delta(k) \\
& +\mathrm{SNR}_{r} \exp \left[-2\left(\frac{\pi w_{r} k}{F_{s}^{\prime}}\right)^{2}\right] \cos \left(2 \pi k \frac{F_{d}}{F_{s}^{\prime}}\right),
\end{aligned}
$$

where $F_{s}^{\prime}=2 m / M F_{s}$ is the sampling frequency of $\tilde{z}$, it is shown that $\sigma_{p, r}$ is such as

$$
\sigma_{p, r}^{2}=\frac{4 m}{M^{2}}\left(\Gamma_{m}\left[\frac{\mathrm{SNR}_{r}}{N^{\prime}}\right]^{2}+2 \frac{\mathrm{SNR}_{r}}{N^{\prime}}+1\right),
$$

where $\mathrm{SNR}_{r} / N^{\prime}$ is the SNR of $\tilde{z}_{r}$ and 


$$
\begin{aligned}
\Gamma_{m} & =\left(1+2 \sum_{k=1}^{2 m-1}(1-k / 2 m) \exp \left[-4\left(\frac{\pi \omega_{r} k}{F_{s}^{\prime}}\right)^{2}\right] \cos \left(2 \pi k \frac{F_{d}}{F_{s}^{\prime}}\right)^{2}\right) \\
& \approx \frac{56.42}{\omega_{r, \mathrm{MHz}}} \frac{m}{128}\left(1-\frac{0.14}{\omega_{r, \mathrm{MHz}}^{2}}\right) .
\end{aligned}
$$

The variance of $\hat{p}$ if it is derived from the average of $N_{a}$ independent range gates, is:

$$
\begin{aligned}
\sigma_{p, N_{a}}^{2} & =\frac{4 m}{M^{2} N_{a}^{2}} \sum_{r=1}^{N_{a}}\left\langle 1+2 \mathrm{SNR}_{r} / N^{\prime}+\Gamma_{m}\left(\mathrm{SNR}_{r} / N^{\prime}\right)^{2}\right\rangle \\
& =\frac{1}{m N_{a}}\left[N^{\prime 2}+2 N^{\prime}\left\langle\overline{\mathrm{SNR}_{r}}\right\rangle+\left\langle\overline{\Gamma_{m}}\right\rangle\left\langle\overline{\mathrm{SNR}_{r}^{2}}\right\rangle\right] .
\end{aligned}
$$

This is expected to underestimate the variance since the spatial correlations of the range-gate parameters $\left(\mathrm{SNR}_{r}\right.$ and to a lesser extent $w_{r}$ ) have been neglected. Still, Fig. 14 shows a good agreement between the variances derived from the ISOSIM data (Eq. 37) and the theoretical calculations (Eq. 45). Results are shown for SNR estimated from the full bandwidth $(\mathrm{m}$ $=128)$ and a narrow bandwidth of $12 \mathrm{MHz}(m=8)$. The $12 \mathrm{MHz}$ bandwidth $(m=8)$ is not a good SNR estimator at altitudes higher than $8 \mathrm{~km}$ (layers 12-17). It is likely because, at these altitudes, $m=12$ is too small a window. However, improving this issue is outside the scope of this study. The consistency between
ISOSIM and the theoretical results is positive for the validation of ISOSIM implementation.

The typical SNR values to get $\sqrt{N_{a}} \times \widehat{\mathrm{SNR}}=0.08$ are between $10^{-3}$ (highest altitudes) and $2.5 \times 10^{-3}$ (lowest altitudes). For such SNR's, the errors on $\sqrt{N_{a}}$ $\times$ SNR are dominated by the detector noise and are independent of the number of accumulated spectra $\left(N_{a}\right)$. Standard deviations near 0.02 are found for the 12 $\mathrm{MHz}$ bandwidth estimates which are consistent with the SNR estimate precision assumed for defining the good-retrievals selection criterion.

\section{Appendix 3: Complementary notations and definitions}

As discussed by (Frehlich and Yadlowsky 1994), the number of photoelectrons $\phi$ coherently detected by range gate is more appropriate than the SNR to characterize the retrieval performances. For a real-valued signal and no range-gates accumulation, it is:

$$
\phi=\frac{M}{2} \mathrm{SNR}
$$
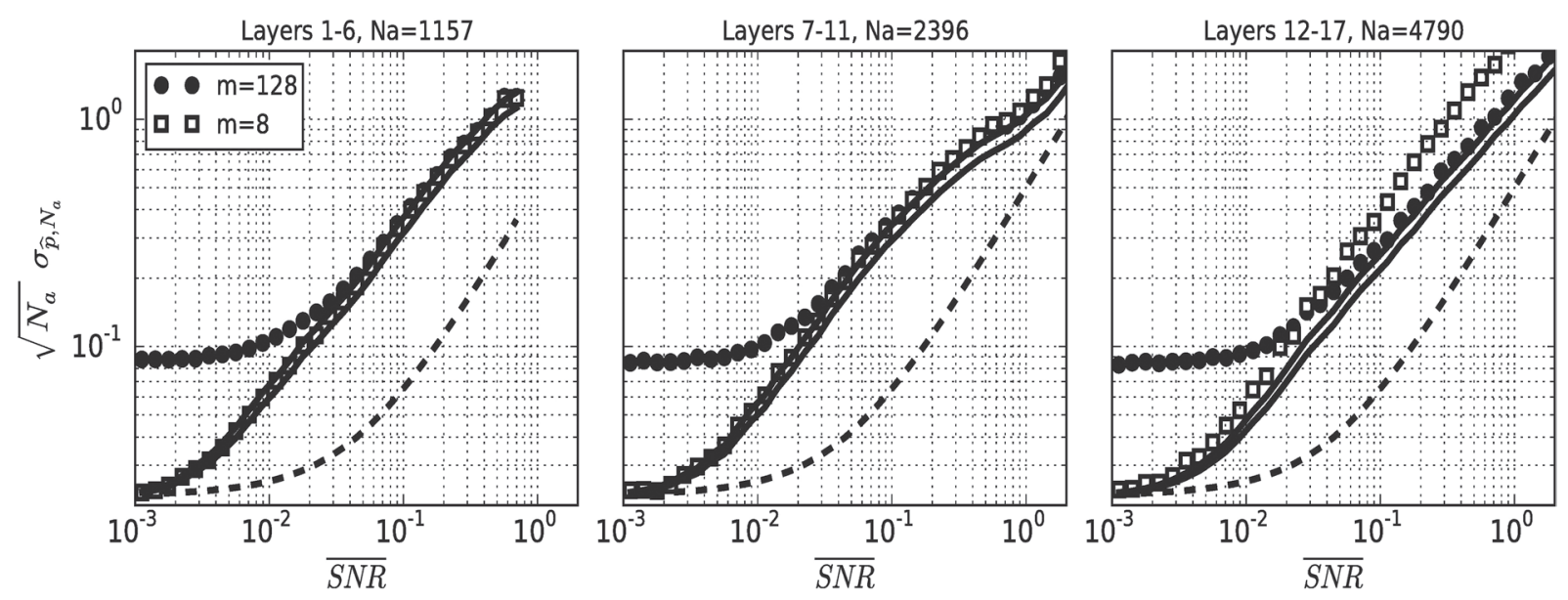

Fig. 14. Standard-deviations of the single-shot SNR estimates (Eq. 37) with respect to the average SNR $(\overline{\mathrm{SNR}})$ for 3 altitude ranges: $0.1-3 \mathrm{~km}$ (layers 1-6), 3-8 km (layers 7-11) and 8-18 km (layers 12-17). The circles and the squares show the results for $m=128$ (full bandwidth) and $m=8$, respectively. The full lines show the theoretical results (Eq. 45) for $m=8$, and $\omega_{r}=1.5 \mathrm{MHz}$ (upper lines) and $3 \mathrm{MHz}$ (lower lines). The dashed lines are the theoretical results for an homogeneous atmosphere $\left(\overline{\mathrm{SNR}_{r}^{2}}=\overline{\mathrm{SNR}}_{r}^{2}\right)$. 
which is simply obtained by multiplying the righthand side of Eq. (4) by $M$ and using $M / B=2 M T_{s}$ for real-valued signals. If the sampling time interval $\left(T_{s}\right.$ $\left.=1 / F_{s}\right)$ is changed but the observation time $\left(M T_{s}\right)$ is fixed, the SNR changes proportionally to $T_{s}$ but $\phi$ remains the same. The spectrum characteristics near the line also remain the same and the frequency retrieval precision should then not change. Therefore, $\phi$ is more appropriate to characterize the retrieval performances.

Note that, since in this study the sampling frequency and the range gate size are fixed, the signal intensity is simply characterized with SNR and not with $\phi$. We need to include $\sqrt{N_{a}}$ when considering rangegates accumulation.

The narrow-range spectrum (Eq. 8) is related to the time-domain signal autocorrelation function $R_{\mathrm{zz}}$ as

$$
\left\langle\hat{P}_{k, M}\left(F_{d}\right)\right\rangle=2 \operatorname{Re}\left\{\operatorname{FFT}\left(R_{\mathrm{zz}}\right)\right\}_{k}-R_{\mathrm{zz}}(0),
$$

where $z$ is signal defined in Eq. (7) and $R_{\mathrm{zz}}(0)=\sigma_{z}^{2}=$ $(1+\mathrm{SNR})$. The correlation time $\tau_{c}$ (s) induced by the Gaussian laser pulse is given by the 1/e FWHM of the envelop of the function $R_{\mathrm{zz}}(i)-\delta(i)$ :

$$
\tau_{c}=\frac{2 \tau}{\sqrt{\ln 2}} \approx 2.402 \tau,
$$

where $\tau$ is the FWHM of the pulse power profile $\left(A^{2}(t)\right.$ in Eq. 4). It is related to the power spectrum standard deviation $\omega_{p}$ (Eq. 9) as:

$$
\tau=\frac{\sqrt{\ln 2 / 2}}{\pi w_{p}}=0.187 w_{p}^{-1} .
$$

A useful parameter often found in other studies is (Frehlich and Yadlowsky 1994):

$$
\Omega=w_{p} \frac{M}{F_{s}}=\frac{\sqrt{2}}{\pi} M_{s},
$$

where $M_{s}=\frac{M T_{s}}{\tau_{c}}$ is the number of independent speckles per range gate. Here $T_{s}=2.5 \mathrm{~ns}, \tau=200 \mathrm{~ns}\left(\tau_{c}=\right.$ $480 \mathrm{~ns}), w_{p}=0.937 \mathrm{MHz}$ and for a range gate of $M=$ 256 samples, $M_{s}=1.33$ and $\omega=0.60$.

\section{Acknowledgment}

We would like to thank the anonymous reviewers for their valuable comments for improving the manuscript. Part of this research was supported by JSPS KAKENHI Grant Number 15K06129 and 15K05293.

\section{References}

Baker, W. E., G. D. Emmitt, F. Robertson, R. M. Atlas, J. E. Molinari, D. A. Bowdle, J. Paegle, R. M. Hardesty, M. J. Post, R. T. Menzies, T. N. Krishnamurti, R. A. Brown, J. R. Anderson, A. C. Lorenc, and J. McElroy, 1995: Lidar-measured winds from space: A key component for weather and climate prediction. Bull. Amer. Meteor. Soc., 76, 869-888.

Baker, W. E., R. Atlas, C. Cardinali, A. Clement, G. D. Emmitt, B. M. Gentry, R. M. Hardesty, E. Källén, M. J. Kavaya, R. Langland, Z. Ma, M. Masutani, W. McCarty, R. B. Pierce, Z. Pu, L. P. Riishojgaard, J. Ryan, S. Tucker, M. Weissmann, and J. G. Yoe, 2014: Lidar-measured wind profiles: The missing link in the global observing system. Bull. Amer. Meteor. Soc., 95, 543-564.

Baron, P., D. P. Murtagh, J. Urban, H. Sagawa, S. Ochiai, Y. Kasai, K. Kikuchi, F. Khosrawi, H. Körnich, S. Mizobuchi, K. Sagi, and M. Yasui, 2013: Observation of horizontal winds in the middle-atmosphere between $30^{\circ} \mathrm{S}$ and $55^{\circ} \mathrm{N}$ during the northern winter 2009-2010. Atmos. Chem. Phys., 13, 6049-6064.

Becker, J. J., D. T. Sandwell, W. H. F. Smith, J. Braud, B. Binder, J. Depner, D. Fabre, J. Factor, S. Ingalls, S.-H. Kim, R. Ladner, K. Marks, S. Nelson, A. Pharaoh, R. Trimmer, J. Von Rosenberg, G. Wallace, and P. Weatherall, 2009: Global bathymetry and elevation data at 30 arc seconds resolution: SRTM30 PLUS. Mar. Geod., 32, 355-371.

Bruneau, D., F. Gibert, P. H. Flamant, and J. Pelon, 2006: Complementary study of differential absorption lidar optimization in direct and heterodyne detections. Appl. Opt., 45, 4898-4908.

Cesana, G., and H. Chepfer, 2012: How well do climate models simulate cloud vertical structure? A comparison between CALIPSO-GOCCP satellite observations and CMIP5 models. Geophys. Res. Lett., 39, L20803, doi:10.1029/2012GL053153.

Cesana, G., and H. Chepfer, 2013: Evaluation of the cloud thermodynamic phase in a climate model using CALIPSO-GOCCP. J. Geophys. Res., 118, 7922-7937.

Chepfer, H., G. Cesana, D. Winker, B. Getzewich, M. Vaughan, and Z. Liu, 2012: Comparison of two different cloud climatologies derived from CALIOP-attenuated backscattered measurements (level 1): The CALIPSOST and the CALIPSO-GOCCP. J. Atmos. Oceanic Technol., 30, 725-744.

Chin, M., P. Ginoux, S. Kinne, O. Torres, B. N. Holben, B. N. Duncan, R. V. Martin, J. A. Logan, A. Higurashi, and T. Nakajima, 2002: Tropospheric aerosol optical thickness from the GOCART model and comparisons with satellite and sun photometer measurements. $J$. Atmos. Sci., 59, 461-483.

Dabas, A., P. H. Flamant, and P. Salamitou, 1994: Characterization of pulsed coherent Doppler lidar with the 
speckle effect. Appl. Opt., 33, 6524-6532.

Dabas, A. M., P. Drobinski, and P. H. Flamant, 2000: Velocity biases of adaptive filter estimates in heterodyne Doppler lidar measurements. J. Atmos. Oceanic Technol., 17, 1189-1202.

Emmitt, G. D., 2001: Feasibility and science merits of a hybrid technology DWL. Proceedings of 11th Coherent Laser Radar Conference, 19-22.

Frehlich, R., 1997: Effects of wind turbulence on coherent Doppler lidar performance. J. Atmos. Oceanic Technol., 14, 54-75.

Frehlich, R., 2000: Simulation of coherent Doppler lidar performance for space-based platforms. J. Appl. Meteor, 39, 245-262.

Frehlich, R., 2001: Errors for space-based Doppler lidar wind measurements: Definition, performance, and verification. J. Atmos. Oceanic Technol., 18, 17491772 .

Frehlich, R., 2004: Velocity error for coherent Doppler lidar with pulse accumulation. J. Atmos. Oceanic Technol., 21, 905-920.

Frehlich, R., and R. Sharman, 2010: Climatology of velocity and temperature turbulence statistics determined from rawinsonde and ACARS/AMDAR data. J. Appl. Meteor. Climatol., 49, 1149-1169.

Frehlich, R. G., and M. J. Yadlowsky, 1994: Performance of mean-frequency estimators for Doppler radar and lidar. J. Atmos. Oceanic Technol., 11, 1217-1230.

Henderson, S. W., 2013: Review of fundamental characteristics of coherent and direct detection Doppler receivers and implications to wind lidar system design. 17th Coherent Laser Radar Conference, Barcelona, Spain.

Henderson, S. W., P. Gatt, D. Rees, and R. M. Huffaker, 2005: Wind lidar. Laser Remote Sensing. Fujii, T., and T. Fukuchi (eds.), Tayloy and Francis Group, 469-722.

Hess, M., P. Koepke, and I. Schult, 1998: Optical properties of aerosols and clouds: The software package OPAC. Bull. Amer. Meteor. Soc., 79, 831-844.

Ishii, S., K. Mizutani, H. Fukuoka, T. Ishikawa, B. Philippe, H. Iwai, T. Aoki, T. Itabe, A. Sato, and K. Asai, 2010: Coherent $2 \mu \mathrm{m}$ differential absorption and wind lidar with conductively cooled laser and two-axis scanning device. Appl. Opt., 49, 1809-1817.

Ishii, S., T. Iwasaki, M. Sato, R. Oki, K. Okamoto, T. Ishibashi, P. Baron, and T. Nishizawa, 2012: Future Doppler lidar wind measurement from space in Japan. Proc. SPIE 8529, Remote Sensing and Modeling of the Atmosphere, Oceans, and Interactions IV, 85290A, doi:10.1117/12.977283.

Ishii, S., K. Okamoto, P. Baron, T. Kubota, Y. Satoh, D. Sakaizawa, T. Ishibashi, T. Y. Tanaka, K. Yamashita, S. Ochiai, K. Gamo, M. Yasui, R. Oki, M. Satoh, and T. Iwasaki, 2016: Measurement performance assessment of future space-borne Doppler wind lidar for numerical weather prediction. SOLA, 12, 55-59.
Ishii, S., P. Baron, M. Aoki, K. Mizutani, M. Yasui, S. Ochiai, A. Sato, Y. Satoh, T. Kubota, D. Sakaizawa, R. Oki, K. Okamoto, T. Ishibashi, T. Tanaka, T. T. Sekiyama, T. Maki, K. Yamashita, T. Nishizawa, M. Satoh, and T. Iwasaki, 2017: Feasibility study for future space-borne coherent Doppler wind lidar, Part 1: Instrumental overview for Global wind profile observation. J. Meteor. Soc. Japan, 95, 301-317.

Iwai, H., S. Ishii, R. Oda, K. Mizutani, S. Sekizawa, and Y. Murayama, 2013: Performance and technique of coherent $2-\mu \mathrm{m}$ differential absorption and wind lidar for wind measurement. J. Atmos. Oceanic Technol., 30, 429-449.

Kawasaki, H., H. Kohata, K. Konoue, Y. Satoh, and S. Imamura, 2013: Development of the Super Low Altitude Test satellite and thermal control. Proceedings of the 29th International Symposium on Space Technology and Science (ISTS), 2013-r-27.

Kiemle, C., M. Quatrevalet, G. Ehret, A. Amediek, A. Fix, and M. Wirth, 2011: Sensitivity studies for a spacebased methane lidar mission. Atmos. Mea. Tech., 4, 2195-2211.

Matsumoto, S., T. Iwata, H. Kawai, T. Sekicuchi, I. Higashino, K. Noguchi, K. Sato, and Y. Torikai, 2013: Technical challenges and solutions to precision autonomous star tracker for agile spacecraft. J. Space Tech. Sci., 28, 57-66.

Okamoto, K., S. Ishii, P. Baron, T. Ishibashi, and T. Tanaka, 2010: Observing Simulation System Experiment (OSSE) of Spaceborne Doppler Wind Lidar. CAS/ JSC WGNE Res. Activ. Atmos. Oceanic Model., 44, $1.15-1.16$.

Okamoto, K., S. Ishii, P. Baron, T. Ishibashi, and T. Tanaka, 2017: Feasibility study for future Space-borne Coherent Doppler Wind Lidar, Part 3: Initial evaluation using the Operational Global Data Assimilation System. J. Meteor. Soc. Japan, submitted.

Rye, B. J., and R. M. Hardesty, 1993: Discrete spectral peak estimation in incoherent backscatter heterodyne lidar. I. Spectral accumulation and the Cramer-Rao lower bound. IEEE Trans. Geosci. Remote Sens., 31, 16-27.

Rye, B. J., and R. M. Hardesty, 1997: Estimate optimization parameters for incoherent backscatter heterodyne lidar. Appl. Opt., 36, 9425-9436.

Salamitou, P., A. Dabas, and P. H. Flamant, 1995: Simulation in the time domain for heterodyne coherent laser radar. Appl. Opt., 34, 499-506.

Sekiyama, T. T., T. Y. Tanaka, A. Shimizu, and T. Miyoshi, 2010: Data assimilation of CALIPSO aerosol observations. Atmos. Chem. Phys., 10, 39-49.

Singh, U. N., M. J. Kavaya, J. Yu, and M. Petros, 2014: Development of 2-micron Doppler wind lidar for NASA 3-D Winds mission. Proc. International Conference on Space Optical Systems and Applications (ICSOS), S5-1, Kobe, Japan.

Stoffelen, A., J. Pailleux, E. Källén, J. M. Vaughan, L. 
Isaksen, P. Flamant, W. Wergen, E. Andersson, H. Schyberg, A. Culoma, R. Meynart, M. Endemann, and P. Ingmann, 2005: The atmospheric dynamics mission for global wind field measurement. Bull. Amer. Meteor. Soc., 86, 73-87.

Tanaka, T. Y., K. Orito, T. T. Sekiyama, K. Shibata, M. Chiba, and H. Tanaka, 2003: MASINGAR, a global tropospheric aerosol chemical transport model coupled with MRI/JMA98 GCM: Model description. Pap. Meteor. Geophys., 53, 119-138.

Uchino, O., T. Sakai, T. Izumi, T. Nagai, I. Morino, A. Yamazaki, M. Deushi, K. Yumimoto, T. Maki, T. Y. Tanaka, T. Akaho, H. Okumura, K. Arai, T. Nakatsuru, T. Matsunaga, and T. Yokota, 2017: Lidar detection of high concentrations of ozone and aerosol transported from northeastern Asia over Saga, Japan. Atmos. Chem. Phys., 17, 1865-1879.

Wessel, P., and W. H. F. Smith, 1996: A global, self-consistent, hierarchical, high-resolution shoreline database. J. Geophys. Res., 101, 8741-8743.

Wu, D., J. Tang, Z. Liu, and Y. Hu, 2013: Simulation of coherent Doppler wind lidar measurement from space based on CALIPSO lidar global aerosol observations. J. Quant. Spectros. Radiat. Transfer, 122, 79-86.

Zrnić, D. S., 1979: Estimation of spectral moments for weather echoes. IEEE Trans. Geosci. Electron., 17, 113-128. 